\title{
Rh Soaked in Poly-Ionic Gel: an Effective Catalyst for Dehydrogenative Silylation of Ketones
}

\author{
Carine Thiot, ${ }^{\#}$ Alain Wagner, ${ }^{* \dagger}$ Charles Mioskowski*,\# \\ Laboratoire de Synthèse Bio-Organique associé au CNRS, Université Louis Pasteur de \\ Strasbourg, Faculté de Pharmacie, 74 route du Rhin, 67401 Illkirch-Graffenstaden, France, \\ and Novalyst Discovery, 23 rue du Loess, 67037 Strasbourg Cedex, France.
}

\section{SUPPORTING INFORMATION}

General Methods. All reactions were carried out under an argon atmosphere. ${ }^{1} \mathrm{H},{ }^{13} \mathrm{C}$ and

${ }^{31} \mathrm{P}$ NMR spectra were recorded on Bruker $200 \mathrm{MHz}$ and $300 \mathrm{MHz}$ spectrometers with $\mathrm{CDCl}_{3}$ as solvent. Chemical shifts are denoted in ppm $(\delta)$ relative to TMS $\left({ }^{1} \mathrm{H},{ }^{13} \mathrm{C}\right)$ or external $\mathrm{H}_{3} \mathrm{PO}_{4}\left({ }^{31} \mathrm{P}\right)$. The gel-phase samples were prepared in $\mathrm{CDCl}_{3}$. For gel-phase ${ }^{31} \mathrm{P}$ experiments, a pulse length of $18.5 \mu \mathrm{s}\left(30^{\circ}\right.$ pulse) was typically used, with a 2.5 s recycle delay, and a 0.32 s acquisition time. Gas chromatography (GC) was performed on a Thermo Finnigan Focus. Infrared spectra of chloroform solutions were recorded on KRS-5 disks using a Perkin Elmer 2000 FTIR spectrophotometer. Solvents were dried and distilled under argon from sodium and benzophenone before use. Chloroform- $d_{1}$ used for NMR measurements was dried extensively over $5 \AA$ molecular sieves. Merrifield resins were purchased from Senn Chemicals (1\% cross-linked with divinylbenzene, 200-400mesh, 1.58 and $2.56 \mathrm{mmol} / \mathrm{g}$ ). Ketones, $\mathrm{BF}_{3} . \mathrm{Et}_{2} \mathrm{O}, \mathrm{Yb}(\mathrm{OTf})_{3}$ and $p$-anisidine were commercially available from Aldrich or Lancaster and used as received. Ketone $\mathbf{3 w}$ was prepared from commercially available acetol and MEMCl. Diphenylsilane (Acros) was stored under an argon atmosphere. $[\mathrm{Rh}(\mathrm{cod}) \mathrm{Cl}]_{2}$ and Wilkinson's catalyst were purchased from Alfa Aesar and used directly. Benzaldehyde was distilled before used. Elemental analysis were carried out by the "Service Central d'Analyse du CNRS" in Vernaison, France. 


\section{Preparation of poly-ionic gel-soaked rhodium catalyst 1.}

Poly-ionic gel. ${ }^{1}$ In a $100 \mathrm{~mL}$ scintillation vial was added the Merrifield type resin (5 g, 7.91 mmol, $2.56 \mathrm{mmol} \mathrm{Cl} / \mathrm{g}$ ), followed by a 1:1 mixture of toluene:acetonitrile $(50 \mathrm{~mL})$. The vial was shaken for $15 \mathrm{~min}$, then triethylamine (17.8 mL, $128.00 \mathrm{mmol}, 10$ equiv) was added and allowed to agitate at $80^{\circ} \mathrm{C}$ for 3 days. The mixture was poured into a fritted funnel and the resin was washed successively with $\mathrm{MeOH} / \mathrm{CH}_{2} \mathrm{Cl}_{2}(3 \times 50 \mathrm{~mL})$ and $\mathrm{Et}_{2} \mathrm{O}(2 \times 50 \mathrm{~mL})$. The resin was transferred into a vial and dried under vacuum to give the poly-ionic gel (5.82 $\mathrm{g})$. Microanalysis (mmol/g): $2.06(\mathrm{~N})$.

Sulfonated phosphine poly-ionic gel. Into a $12 \mathrm{~mL}$-Supelco syringe was added the previously prepared poly-ionic gel (300 mg, $0.618 \mathrm{mmol}, 2.06 \mathrm{mmol} \mathrm{N} / \mathrm{g}$ ) and DMF (4 mL). The resin was then drained, then a solution of triphenylphosphine-3,3',3' -trisulfonic acid trisodium salt ( $1.37 \mathrm{~g}, 2.41 \mathrm{mmol}, 3.9$ equiv in $21 \mathrm{~mL}$ of a $4: 1$ mixture of DMF- $\mathrm{H}_{2} \mathrm{O}$ ) was added in 3 times ( $3 \times 7 \mathrm{~mL}$ ) into the syringe. Between each anionic exchange, the syringe was shaken at room temperature for $1 \mathrm{~h}$. Finally, the beads were drained and successively washed with DMF (3x $4 \mathrm{~mL}), \mathrm{CH}_{2} \mathrm{Cl}_{2}(3 \mathrm{x} 4 \mathrm{~mL})$ and $\mathrm{Et}_{2} \mathrm{O}(2 \mathrm{x} 4 \mathrm{~mL})$. Air was used to dry the beads before being transferred to a vial and dried under vacuum to afford $322 \mathrm{mg}$ of the sulfonated phosphine poly-ionic gel. Microanalysis $(\mathrm{mmol} / \mathrm{g}): 1.51(\mathrm{~N}), 0.54(\mathrm{P}) . \mathrm{IR}\left(\mathrm{KBr}, \mathrm{cm}^{-1}\right): 615$ (vC-S), 672 (vS-O), 778 (vP-C), 1090 (vS=O), 1227. Gel-phase ${ }^{31} \mathrm{P}\left\{{ }^{1} \mathrm{H}\right\}$ NMR: $\delta-3.5$.

Poly-ionic gel-soaked rhodium catalyst 1. In a $25 \mathrm{~mL}$ scintillation vial, was added the previously prepared sulfonated phosphine poly-ionic gel $(120 \mathrm{mg}, 0.064 \mathrm{mmol}, 0.54 \mathrm{mmol}$ $\mathrm{P} / \mathrm{g})$ to a solution of dimeric $[\mathrm{Rh}(\mathrm{cod}) \mathrm{Cl}]_{2}$ precursor $(3.2 \mathrm{mg}, 0.006 \mathrm{mmol}, 0.1$ equiv in $4 \mathrm{~mL}$ of $\mathrm{CH}_{2} \mathrm{Cl}_{2}$ ). The vial was shaken for at $45^{\circ} \mathrm{C}$ for $6 \mathrm{~h}$. The mixture was poured into a fritted funnel and the resin was washed with $\mathrm{CH}_{2} \mathrm{Cl}_{2}(3 \times 5 \mathrm{~mL})$ and $\mathrm{Et}_{2} \mathrm{O}(2 \times 5 \mathrm{~mL})$. The resin was transferred into a vial and dried under vacuum to give the poly-ionic gel-soaked rhodium 1 (135 mg). Microanalysis (mmol/g): N (1.62), P (0.57), Rh (0.16). Far IR (nujol, $\mathrm{cm}^{-1}$ ): 299 (vRh-Cl). Gel-phase ${ }^{31} \mathrm{P}\left\{{ }^{1} \mathrm{H}\right\}$ NMR: $\delta 33.7$.

\section{Preparation of poly-ionic gel-soaked rhodium catalyst 2.}

Poly-ionic gel. In a $100 \mathrm{~mL}$ scintillation vial was added the Merrifield type resin (5 g, 7.91 mmol, $1.58 \mathrm{mmol} \mathrm{Cl} / \mathrm{g}$ ), followed by a 1:1 mixture of toluene:acetonitrile $(50 \mathrm{~mL})$. The vial was shaken for $15 \mathrm{~min}$, then triethylamine $(11 \mathrm{~mL}, 79.12 \mathrm{mmol}, 10$ equiv) was added and 
allowed to agitate at $80^{\circ} \mathrm{C}$ for 3 days. The mixture was poured into a fritted funnel and the resin was washed successively with $\mathrm{MeOH} / \mathrm{CH}_{2} \mathrm{Cl}_{2}(3 \times 50 \mathrm{~mL})$ and $\mathrm{Et}_{2} \mathrm{O}(2 \mathrm{x} 50 \mathrm{~mL})$. The resin was transferred into a vial and dried under vacuum to give the poly-ionic gel (5.47 $\mathrm{g}$ ). Microanalysis (mmol/g): $1.32(\mathrm{~N})$.

Poly-ionic gel-soaked rhodium catalyst 2. In a $25 \mathrm{~mL}$ scintillation vial was added the previously prepared poly-ionic gel $(100 \mathrm{mg}, 0.132 \mathrm{mmol}, 1.32 \mathrm{mmol} \mathrm{N} / \mathrm{g}$ ) to a solution of Wilkinson's precursor (252 $\mathrm{mg}, 0.264 \mathrm{mmol}, 2$ equiv in $3 \mathrm{~mL}$ of $\mathrm{CH}_{2} \mathrm{Cl}_{2}$ ). The vial was shaken at $45^{\circ} \mathrm{C}$ for $6 \mathrm{~h}$. The mixture was poured into a fritted funnel and the resin was washed with $\mathrm{CH}_{2} \mathrm{Cl}_{2}(3 \times 5 \mathrm{~mL})$ and $\mathrm{Et}_{2} \mathrm{O}(2 \times 5 \mathrm{~mL})$. The resin was transferred into a vial and dried under vacuum to give rhodium catalyst $2(120 \mathrm{mg})$. Microanalysis $(\mathrm{mmol} / \mathrm{g}): 1.36(\mathrm{~N}), 0.21$ (Rh). Far IR (nujol, $\mathrm{cm}^{-1}$ ): 312 (vRh-Cl). Gel-phase ${ }^{31} \mathrm{P}\left\{{ }^{1} \mathrm{H}\right\}$ NMR: $\delta$ 29.9.

\section{General Procedure for the Dehydrogenative Silylation of Ketones with Rh catalyst $1 .^{2}$ To} a suspension of Rh catalyst $1(13.1 \mathrm{mg}, 2.09 \mu \mathrm{mol}, 0.16 \mathrm{mmol} \mathrm{Rh} / \mathrm{g})$ in dry $\mathrm{CH}_{2} \mathrm{Cl}_{2}(1 \mathrm{~mL})$ was added a solution of acetophenone $3 \mathbf{a}\left(53 \mathrm{mg}, 0.44 \mathrm{mmol}, 1\right.$ equiv in $1 \mathrm{~mL}$ of dry $\left.\mathrm{CH}_{2} \mathrm{Cl}_{2}\right)$. The resulting mixture was stirred at room temperature for $10 \mathrm{~min}$, and then cooled to $0{ }^{\circ} \mathrm{C}$. A solution of diphenylsilane $4\left(163 \mu \mathrm{L}, 0.88 \mathrm{mmol}, 2\right.$ equiv in $2 \mathrm{~mL}$ of dry $\left.\mathrm{CH}_{2} \mathrm{Cl}_{2}\right)$ was added dropwise. The stirred reaction solution was maintained for $1 \mathrm{~h}$ at $0^{\circ} \mathrm{C}$ and then was allowed to stir at room temperature for $6 \mathrm{~h}$. The reaction mixture was filtered-off through celite, and concentrated under reduced pressure. \% conversion and ratios 5a:6a were measured by ${ }^{1} \mathrm{H}$ NMR spectroscopy of the crude reaction mixture, using benzofuran as an internal standard when indicated. Aromatic signals of the diphenylsilyl enols 5 were not indicated in the below ${ }^{1} \mathrm{H}$ NMR data because of the overlap of the aromatic protons of 4 .

Table 2, entry 1: 1-(Diphenylsiloxy)-1-(phenyl)ethene 5a. Oil. Conv.(\%): 95, 5a:6a = 84:16. ${ }^{1} \mathrm{H}$ NMR: $\delta 4.55(\mathrm{~d}, J=2.4 \mathrm{~Hz}, 1 \mathrm{H}), 4.96(\mathrm{~d}, J=2.4 \mathrm{~Hz}, 1 \mathrm{H}), 5.64$ (s, 1H). Spectral datal were in accordance with those previously reported. ${ }^{3}$

Table 2, entry 2: 1-(Diphenylsiloxy)-1-(4-methylphenyl)ethene 5b. Oil. Conv.(\%): 61, 5b:6b = 48:52. ${ }^{1} \mathrm{H}$ NMR: $\delta 2.40(\mathrm{~s}, 3 \mathrm{H}), 4.53(\mathrm{~d}, J=2.5 \mathrm{~Hz}, 1 \mathrm{H}), 4.94(\mathrm{~d}, J=2.5 \mathrm{~Hz}, 1 \mathrm{H})$, $5.78(\mathrm{~s}, 1 \mathrm{H})$. 
Table 2, entry 3: 1-(Diphenylsiloxy)-1-(4-methoxyphenyl)ethene 5c. Oil. Conv.(\%): 65, 5c:6c $=0: 100 .{ }^{1} \mathrm{H}$ NMR: $\delta 3.93(\mathrm{~s}, 3 \mathrm{H}), 4.52(\mathrm{~d}, J=2.5 \mathrm{~Hz}, 1 \mathrm{H}), 4.91(\mathrm{~d}, J=2.5 \mathrm{~Hz}, 1 \mathrm{H})$, $5.81(\mathrm{~s}, 1 \mathrm{H})$.

Table 2, entry 4: 1-(4-Bromophenyl)-1-(diphenylsiloxy)ethene 5d. Oil. Conv.(\%): 96, 5d:6d = 91:9. ${ }^{1} \mathrm{H}$ NMR: $\delta 4.59(\mathrm{~d}, J=2.2 \mathrm{~Hz}, 1 \mathrm{H}), 4.97(\mathrm{~d}, J=2.2 \mathrm{~Hz}, 1 \mathrm{H}), 5.76(\mathrm{~s}, 1 \mathrm{H})$.

Table 2, entry 5: 1-(Diphenylsiloxy)-1-(4-trifluoromethanephenyl)ethene 5e. Oil. Conv.(\%): 99, 5e:6e = 98:2. ${ }^{1} \mathrm{H}$ NMR: $\delta 4.71(\mathrm{~d}, J=2.7 \mathrm{~Hz}, 1 \mathrm{H}), 5.10(\mathrm{~d}, J=2.7 \mathrm{~Hz}, 1 \mathrm{H})$, $5.81(\mathrm{~s}, 1 \mathrm{H})$.

Table 2, entry 6: 1-(Diphenylsiloxy)-1-(4-ethylesterphenyl)ethene 5f. Oil. Conv.(\%): 97, 5f:6f = 99:1. ${ }^{1} \mathrm{H}$ NMR: $\delta 1.45(\mathrm{t}, J=7.1 \mathrm{~Hz}, 3 \mathrm{H}), 4.45(\mathrm{q}, J=7.1 \mathrm{~Hz}, 2 \mathrm{H}), 4.71(\mathrm{~d}, J=2.2$ $\mathrm{Hz}, 1 \mathrm{H}), 5.12$ (d, $J=2.2 \mathrm{~Hz}, 1 \mathrm{H}), 5.81$ (s, 1H). (See ${ }^{1} \mathrm{H}$ NMR spectrum below).

To ensure that no side product (except the silylalkyl ether 6f) was formed, the reaction mixture containing $\mathbf{5 f}$ was hydrolyzed. A solution of $1 \mathrm{~mL}$ of $\mathrm{MeOH}$ containing $1 \% \mathrm{TsOH}$ was added dropwise, and the reaction mixture was stirred for $20 \mathrm{~min}$ at room temperature. ${ }^{1} \mathrm{H}$ NMR of the crude reaction mixture gave a 96:4 ratio of starting ketone $\mathbf{3 f} /$ reduced ketone. Oil. ${ }^{1} \mathrm{H}$ NMR (300 MHz, $\left.\mathrm{CDCl}_{3}\right): 1.43(\mathrm{t}, J=7.4 \mathrm{~Hz}, 3 \mathrm{H}), 2.64$ (s, 3H), 4.46 (q, $\left.J=7.4 \mathrm{~Hz}, 2 \mathrm{H}\right)$, 8.00-8.03 (d, $J=8.7 \mathrm{~Hz}, 2 \mathrm{H}), 8.13-8.16(\mathrm{~d}, J=8.7 \mathrm{~Hz}, 2 \mathrm{H})$ for ethyl 4-acetylbenzoate $3 \mathbf{f}$. $1.41(\mathrm{t}, J=7.1 \mathrm{~Hz}, 3 \mathrm{H}), 1.51(\mathrm{~d}, J=6.5 \mathrm{~Hz}, 3 \mathrm{H}), 4.40(\mathrm{q}, J=7.1 \mathrm{~Hz}, 2 \mathrm{H}), 4.97$ (q, $J=6.5 \mathrm{~Hz}$, $1 \mathrm{H})$, 7.93-7.96 (d, $J=8.1 \mathrm{~Hz}, 2 \mathrm{H}), 8.03-8.06(\mathrm{~d}, J=8.1 \mathrm{~Hz}, 2 \mathrm{H})$ for 1-(4ethylesterphenyl)ethan-1-ol. (See ${ }^{1} \mathrm{H}$ NMR spectrum below).

Table 2, entry 7: 1-(Diphenylsiloxy)-1-(4-trifluoromethanesulfonatephenyl)ethene 5g. Oil. Conv.(\%): 98, 5g:6g = 99:1. ${ }^{1} \mathrm{H}$ NMR: $\delta 4.67(\mathrm{~d}, J=2.8 \mathrm{~Hz}, 1 \mathrm{H}), 5.01(\mathrm{~d}, J=2.8 \mathrm{~Hz}, 1 \mathrm{H})$, $5.79(\mathrm{~s}, 1 \mathrm{H})$.

Table 2, entry 8: 1-(Diphenylsiloxy)-1-(naphtyl)ethene 5h. Oil. Conv.(\%): 96, 5h:6h = 77:23. ${ }^{1} \mathrm{H}$ NMR: $\delta 4.78(\mathrm{~d}, J=2.6 \mathrm{~Hz}, 1 \mathrm{H}), 5.22(\mathrm{~d}, J=2.6 \mathrm{~Hz}, 1 \mathrm{H}), 5.92(\mathrm{~s}, 1 \mathrm{H})$.

Yields and ratios were calculated by ${ }^{1} \mathrm{H}$ NMR spectroscopy using benzofuran as an internal standard. 
Table 3, entry 1: (Z)-1-(Diphenylsiloxy)-1-(phenyl)pent-1-ene 5i. ${ }^{4}$ Oil. Yield (\%): 56. ${ }^{1} \mathrm{H}$ NMR: $\delta 0.98$ (t, $J=7.1 \mathrm{~Hz}, 3 \mathrm{H}), 1.42$ (sextet, $J=7.1 \mathrm{~Hz}, 2 \mathrm{H}), 2.28$ (q, $J=7.1 \mathrm{~Hz}, 2 \mathrm{H}), 5.42$ $(\mathrm{t}, J=7.1 \mathrm{~Hz}, 1 \mathrm{H}), 5.71(\mathrm{~s}, 1 \mathrm{H})$.

Table 3, entry 3: 1-(Diphenylsiloxy)-3,4-dihydronaphtalene $5 \mathbf{k}^{5}$ Oil. Yield (\%): $75 .{ }^{1} \mathrm{H}$ NMR: $\delta 2.32(\mathrm{td}, J=4.5,8.2 \mathrm{~Hz}, 2 \mathrm{H}), 2.79(\mathrm{t}, J=8.2 \mathrm{~Hz}, 2 \mathrm{H}), 5.30(\mathrm{t}, J=4.5 \mathrm{~Hz}, 1 \mathrm{H}), 5.76$ $(\mathrm{s}, 1 \mathrm{H})$.

Table 3, entry 4: 2-(Diphenylsiloxy)-4,4-dimethyl-pent-1-ene 5l. Oil. Yield (\%): 91, 51:5l' = 90:10. ${ }^{1} \mathrm{H}$ NMR: $\delta 1.17$ (s, 9H), 2.21 (s, 2H), 4.23 (d, $\left.J=1.3 \mathrm{~Hz}, 1 \mathrm{H}\right), 4.43(\mathrm{~d}, J=1.3 \mathrm{~Hz}$, $1 \mathrm{H}), 5.81(\mathrm{~s}, 1 \mathrm{H})$.

Table 3, entry 4: 2-(Diphenylsiloxy)-4,4-dimethyl-pent-2-ene 5l'.6 Oil. Yield (\%): 91, 5l:5l' $=90: 10 .{ }^{1} \mathrm{H}$ NMR: $\delta 1.09(\mathrm{~s}, 9 \mathrm{H}), 1.98(\mathrm{~s}, 3 \mathrm{H}), 4.55(\mathrm{~s}, 1 \mathrm{H}), 5.86(\mathrm{~s}, 1 \mathrm{H})$.

Table 3, entry 5: 1-(Diphenylsiloxy)-1-cyclohexyl-ethene 5m. ${ }^{7}$ Oil. Yield (\%): 85. ${ }^{1} \mathrm{H}$ NMR: $\delta 4.24(\mathrm{~d}, J=1.5 \mathrm{~Hz}, 1 \mathrm{H}), 4.28(\mathrm{~d}, J=1.5 \mathrm{~Hz}, 1 \mathrm{H}), 5.77(\mathrm{~s}, 1 \mathrm{H})$.

Table 3, entry 6: 2-(Diphenylsiloxy)-3,3-dimethyl-but-1-ene 5n. ${ }^{7}$ Oil. Yield (\%): $98 .{ }^{1} \mathrm{H}$ NMR: $\delta 1.23(\mathrm{~s}, 9 \mathrm{H}), 4.18(\mathrm{~d}, J=1.9 \mathrm{~Hz}, 1 \mathrm{H}), 4.26(\mathrm{~d}, J=1.9 \mathrm{~Hz}, 1 \mathrm{H}), 5.72(\mathrm{~s}, 1 \mathrm{H})$.

Yields and ratios were calculated by ${ }^{1} \mathrm{H}$ NMR spectroscopy using benzofuran as an internal standard.

Table 4, entry 1: 2-(Diphenylsiloxy)-pent-1-ene 5o. ${ }^{8}$ Oil. Yield $(\%): 28$, 5o:5o' $=31: 69 .{ }^{1} \mathbf{H}$ NMR: $\delta .1 .94$ (quin, $J=7.5 \mathrm{~Hz}, 2 \mathrm{H}), 4.15(\mathrm{~d}, J=1.2 \mathrm{~Hz}, 1 \mathrm{H}), 4.23(\mathrm{~d}, J=1.2 \mathrm{~Hz}, 1 \mathrm{H}), 5.61$ $(\mathrm{s}, 1 \mathrm{H})$.

Table 4, entry 1: (Z)-1-(Diphenylsiloxy)-1-methyl-but-1-ene 5o'. ${ }^{8}$ Oil. Yield (\%): 28, 5o:5o' $=31: 69 .{ }^{1} \mathrm{H}$ NMR: $\delta 1.83(\mathrm{t}, J=1.2 \mathrm{~Hz}, 3 \mathrm{H}$ ), 2.13 (dquin, $J=1.2,7.2 \mathrm{~Hz}, 2 \mathrm{H}$ ), 5.57 (br s, 1H), $5.64(\mathrm{~s}, 1 \mathrm{H})$.

Table 4, entry 2: 1-(Diphenylsiloxy)-1-(methoxymethyl)ethene 5p. ${ }^{9}$ Oil. Yield (\%): 88, 5q:5q' = 63:37. ${ }^{1} \mathrm{H}$ NMR: $\delta 3.35$ (s, 3H), $3.91(\mathrm{~d}, J=0.6 \mathrm{~Hz}, 2 \mathrm{H}), 4.45$ (br s, 1H), 4.48 (d, $J=$ $1.5 \mathrm{~Hz}, 1 \mathrm{H}), 5.68(\mathrm{~s}, 1 \mathrm{H})$.

Table 4, entry 2: (E)- 1-(diphenylsiloxy)-1-methyl-2-methoxy-ethene 5p'. Oil. Yield (\%): 88, (E)-5q':(Z)-5q' = 35:65. ${ }^{1} \mathrm{H}$ NMR: $\delta 1.76(\mathrm{~d}, J=1.2 \mathrm{~Hz}, 3 \mathrm{H}), 3.32(\mathrm{~s}, 3 \mathrm{H}), 5.41$ (q, $J=$ $1.2 \mathrm{~Hz}, 1 \mathrm{H}), 5.61(\mathrm{~s}, 1 \mathrm{H})$.

Table 4, entry 2: (Z)-1-(diphenylsiloxy)-1-methyl-2-methoxy-ethene 5p'.9 Oil. Yield (\%): 88, (E)-5q':(Z)-5q' = 35:65. 'H NMR: $\delta 1.88(\mathrm{~d}, J=1.2 \mathrm{~Hz}, 3 \mathrm{H}), 3.45$ (s, 3H), 5.69 (s, 1H), $6.00(\mathrm{q}, J=1.2 \mathrm{~Hz}, 1 \mathrm{H})$. 
Table 4, entry 3: 1,1-(Methylmethoxy)methyl-1-(diphenylsiloxy)ethene 5q. Oil. Yield (\%): 95. ${ }^{1} \mathrm{H}$ NMR: $\delta 1.31(\mathrm{~d}, J=6.4 \mathrm{~Hz}, 3 \mathrm{H}), 3.35(\mathrm{~s}, 3 \mathrm{H}), 3.76(\mathrm{q}, J=6.4 \mathrm{~Hz}, 1 \mathrm{H}), 4.46$ (br s, $1 \mathrm{H}), 4.51(\mathrm{~d}, J=1.5 \mathrm{~Hz}, 1 \mathrm{H}), 5.67(\mathrm{~s}, 1 \mathrm{H})$.

Table 4, entry 4: 1-Dimethoxymethyl-1-(diphenylsiloxy)ethene $5 \mathbf{r} .{ }^{10}$ Oil. Yield (\%): $98 .{ }^{1} \mathrm{H}$ NMR: $\delta 3.34$ (s, 6H), $4.56(\mathrm{~d}, J=1.5 \mathrm{~Hz}, 1 \mathrm{H}), 4.69$ (d, $J=1.5 \mathrm{~Hz}, 1 \mathrm{H}), 4.74$ (br s, 1H), 5.67 $(\mathrm{s}, 1 \mathrm{H})$.

Table 4, entry 5: Methyl-3-(diphenylsiloxy)-but-3-enoate 5s. Oil. Yield (\%): 81, 5s:5s' = 76:24. ${ }^{1} \mathrm{H}$ NMR: $\delta 3.21(\mathrm{~s}, 2 \mathrm{H}), 3.68(\mathrm{~s}, 3 \mathrm{H}), 4.31(\mathrm{~d}, J=1.9 \mathrm{~Hz}, 1 \mathrm{H}), 4.38(\mathrm{~d}, J=1.9 \mathrm{~Hz}$, $1 \mathrm{H}), 5.71(\mathrm{~s}, 1 \mathrm{H})$.

Table 4, entry 5: Methyl-(2Z)-3-(diphenylsiloxy)-but-2-enoate 5s'. Oil. Yield (\%): 81, 5s:5s' = 76:24. ${ }^{1} \mathrm{H}$ NMR: $\delta 2.41$ (s, 3H), 3.66 (s, 3H), 5.39 (br s, 1H), 5.71 (s, 1H).

Table 4, entry 6: Ethyl-3-(diphenylsiloxy)-2-methyl-but-3-enoate 5t. Oil. Yield (\%): 82. ${ }^{1} \mathrm{H}$ NMR: $\delta 1.23(\mathrm{t}, J=7.2 \mathrm{~Hz}, 3 \mathrm{H}), 1.38(\mathrm{~d}, J=7.1 \mathrm{~Hz}, 3 \mathrm{H}), 3.28(\mathrm{q}, J=7.1 \mathrm{~Hz}, 1 \mathrm{H}), 4.14(\mathrm{dq}, J$ $=0.6,7.2 \mathrm{~Hz}, 2 \mathrm{H}), 4.36(\mathrm{~m}, 2 \mathrm{H}), 5.64(\mathrm{~s}, 1 \mathrm{H})$.

Table 4, entry 7: 4-(Diphenylsiloxy)-3-methyl-pent-4-en-2-one 5u. Oil. Yield (\%): 81, 5u:5u" = 74:26. ${ }^{1} \mathrm{H}$ NMR: $\delta 1.25(\mathrm{~d}, J=7.1 \mathrm{~Hz}, 3 \mathrm{H}), 2.18(\mathrm{~s}, 3 \mathrm{H}), 3.25(\mathrm{q}, J=7.1 \mathrm{~Hz}, 1 \mathrm{H})$, $4.33(\mathrm{~d}, J=2.1 \mathrm{~Hz}, 1 \mathrm{H}), 4.39(\mathrm{~d}, J=2.1 \mathrm{~Hz}, 1 \mathrm{H}), 5.63(\mathrm{~s}, 1 \mathrm{H})$.

Table 4, entry 7: Bis-(diphenylsilyl ether enol) 5u'. Oil. Yield (\%): 81, 5u:5u' $=74: 26 .{ }^{1} \mathrm{H}$ NMR: $\delta 1.36(\mathrm{~d}, J=7.1 \mathrm{~Hz}, 3 \mathrm{H}), 3.06(\mathrm{q}, J=7.1 \mathrm{~Hz}, 1 \mathrm{H}), 4.28(\mathrm{~d}, J=1.9 \mathrm{~Hz}, 2 \mathrm{H}), 4.29(\mathrm{~d}, J$ $=1.9 \mathrm{~Hz}, 2 \mathrm{H}), 5.61(\mathrm{~s}, 1 \mathrm{H})$.

Table 4, entry 8: Bis-(diphenylsilyl ether enol) 5u'’. Oil. Yield (\%): 98. 'H NMR: $\delta 1.34$ (d, $J=7.1 \mathrm{~Hz}, 3 \mathrm{H}), 3.10(\mathrm{q}, J=7.1 \mathrm{~Hz}, 1 \mathrm{H}), 4.32(\mathrm{~d}, J=1.8 \mathrm{~Hz}, 2 \mathrm{H}), 4.34(\mathrm{~d}, J=1.8 \mathrm{~Hz}, 2 \mathrm{H})$, $5.66(\mathrm{~s}, 1 \mathrm{H})$.

Table 4, entry 9: 1-(Diphenylsiloxy)-1-(2-methoxyphenyl)ethene 5v. Oil. Yield.(\%): $63 .{ }^{1} \mathrm{H}$ NMR: $\delta 3.82(\mathrm{~s}, 3 \mathrm{H}), 4.84(\mathrm{~d}, J=1.5 \mathrm{~Hz}, 1 \mathrm{H}), 5.11(\mathrm{~d}, J=1.5 \mathrm{~Hz}, 1 \mathrm{H}), 5.73(\mathrm{~s}, 1 \mathrm{H})$

Table S1. Effect of Coordinating Groups on Ketones ${ }^{a}$

\begin{tabular}{|c|c|c|c|c|c|}
\hline \multicolumn{2}{|c|}{3} & $\begin{array}{c}\mathbf{1}(0.4 \mathrm{~mol} \%) \\
\mathrm{Ph}_{2} \mathrm{SiH}_{2} 4 \\
\mathrm{CH}_{2} \mathrm{Cl}_{2}, \mathrm{rt}, 6 \mathrm{~h}\end{array}$ & 5 & $\overbrace{\mathbf{5}^{\prime}}^{\mathrm{OSiHPh}_{2}}+$ & $\underbrace{\mathrm{OSiHPh}_{2}}_{6}$ \\
\hline entry & 3 & $\mathrm{R}_{1}$ & $5+5^{\prime}: 6$ & $\mathbf{5 : 5 ^ { b }}$ & yield $\mathbf{5 + 5}(\%)^{b}$ \\
\hline 1 & 30 & $\mathrm{Me}$ & $34: 66$ & $31: 69(Z)$ & 28 \\
\hline 2 & $3 \mathbf{p}$ & $\mathrm{OMe}$ & $92: 8$ & $63: 37(E / Z: 35 / 65)$ & 88 \\
\hline 3 & $3 w$ & MEM & $95: 5$ & $74: 26(Z)$ & 85 \\
\hline 4 & $3 \mathbf{x}$ & $\mathrm{OSiMe}_{2} \mathrm{tBu}$ & $79: 21$ & $63: 37(Z)$ & 71 \\
\hline 5 & $3 \mathbf{y}$ & $\mathrm{OPh}$ & $65: 35$ & $65: 35(Z)$ & 54 \\
\hline
\end{tabular}


${ }^{a} 3(0.44 \mathrm{mmol}), 4(0.88 \mathrm{mmol})$ was stirred in $\mathrm{CH}_{2} \mathrm{Cl}_{2}(4 \mathrm{~mL})$ in the presence of catalyst $(0.4 \mathrm{~mol} \%$ of metal) at $\mathrm{rt}$ for $6 \mathrm{~h}$ under argon. ${ }^{b}$ Measured by ${ }^{1} \mathrm{H}$ NMR spectroscopy using benzofuran as an internal standard of the crude reaction mixture.

Table S1, entry 3: 1-(Diphenylsiloxy)-1-[[(2-(methoxyethoxy)methoxy]methyl]ethene 5w. Oil. Yield (\%): 85, 5w:5w' = 74:264. ${ }^{1} \mathrm{H}$ NMR: $\delta 3.38(\mathrm{~s}, 3 \mathrm{H}), 3.51-3.54(\mathrm{~m}, 2 \mathrm{H}), 3.67-3.70$ $(\mathrm{m}, 2 \mathrm{H}), 4.03(\mathrm{~s}, 2 \mathrm{H}), 4.40(\mathrm{~d}, J=1.2 \mathrm{~Hz}, 1 \mathrm{H}), 4.43(\mathrm{~s}, 1 \mathrm{H}), 4.70(\mathrm{~s}, 2 \mathrm{H}), 5.61(\mathrm{~s}, 1 \mathrm{H})$.

Table S1, entry 3: (Z)-1-(diphenylsiloxy)-1-methyl-2-[2-(methoxyethoxy)methoxy]-ethene 5w'. Oil. Yield (\%): 85, 5w:5w' = 74:26. ${ }^{1} \mathrm{H}$ NMR: $\delta 1.85(\mathrm{~d}, J=0.9 \mathrm{~Hz}, 3 \mathrm{H}), 3.38(\mathrm{~s}, 3 \mathrm{H})$, 3.45-3.47 (m, 2H), 3.64-3.65 (m, 2H), 4.75 (s, 2H), 5.48 (s, 1H), 6.18 (q, $J=0.9 \mathrm{~Hz}, 1 \mathrm{H})$.

Table S1, entry 4: 1-(Diphenylsiloxy)-1-(tert-butyldimethylsilyloxymethyl)ethene 5x. Oil. Yield (\%): 71, 5x:5x' = 63:37. ${ }^{1} \mathrm{H}$ NMR: $\delta 0.06(\mathrm{~s}, 6 \mathrm{H}), 0.91(\mathrm{~s}, 9 \mathrm{H}), 4.06(\mathrm{~s}, 2 \mathrm{H}), 4.34(\mathrm{~s}$, $1 \mathrm{H}), 4.48(\mathrm{~s}, 1 \mathrm{H}), 5.63(\mathrm{~s}, 1 \mathrm{H})$.

Table S1, entry 4: (Z)-1-(diphenylsiloxy)-1-methyl-2-tert-butyldimethylsilyloxy-ethene 5x'. Oil. Yield (\%): 71, 5x:5x' = 63:37. ${ }^{1} \mathrm{H}$ NMR: $\delta 0.00(\mathrm{~s}, 6 \mathrm{H}), 0.87(\mathrm{~s}, 9 \mathrm{H}), 1.83(\mathrm{~d}, J=0.9$ $\mathrm{Hz}, 3 \mathrm{H}), 5.51$ (s, 1H), 6.17 (q, $J=1.2 \mathrm{~Hz}, 1 \mathrm{H})$.

Table S1, entry 5: 1-(Diphenylsiloxy)-1-(phenoxymethyl)ethene 5y. Oil. Yield (\%): 54, 5y:5y' = 65:35. ${ }^{1} \mathrm{H}$ NMR: $\delta 4.48(\mathrm{~s}, 2 \mathrm{H}), 4.50(\mathrm{~s}, 2 \mathrm{H}), 5.67(\mathrm{~s}, 1 \mathrm{H})$.

Table S1, entry 5: (Z)-1-(diphenylsiloxy)-1-methyl-2-phenoxy-ethene 5y'. Oil. Yield (\%): 54, 5y:5y' = 65:35. ${ }^{1} \mathrm{H}$ NMR: $\delta 1.93(\mathrm{~d}, J=0.9 \mathrm{~Hz}, 3 \mathrm{H}), 5.64(\mathrm{~s}, 1 \mathrm{H}), 6.27$ (q, $J=1.2 \mathrm{~Hz}$, $1 \mathrm{H})$.

Table S2. Effect of Bulky Phosphines (Cone angle) on Selectivity ${ }^{a}$
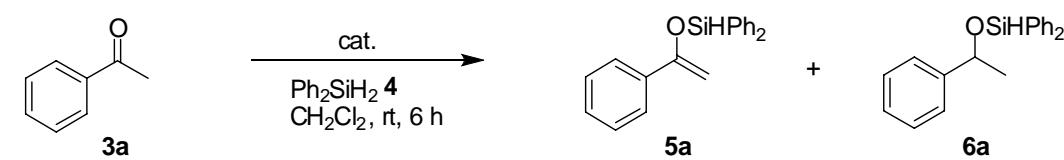

\begin{tabular}{|c|c|c|c|c|}
\hline entry & catalyst & cone angle $\left(^{\circ}\right)$ & $5 a: 6 a$ & convsn $^{b}$ \\
\hline 1 & cat. 1 & - & $84: 16$ & 95 \\
\hline 2 & $\begin{array}{l}{[\mathrm{Rh}(\operatorname{cod}) \mathrm{Cl}]_{2} /} \\
\text { TPPTS } / \mathrm{NBu}_{4} \mathrm{Cl}^{c}\end{array}$ & 170 & $22: 78$ & 77 \\
\hline 3 & PS-PPh & - & $53: 47$ & 74 \\
\hline 4 & $\begin{array}{l}{[\mathrm{Rh}(\operatorname{cod}) \mathrm{Cl}]_{2} /} \\
\mathrm{PPh}_{3}{ }^{d}\end{array}$ & 145 & $1: 99$ & 96 \\
\hline 5 & $\begin{array}{l}{[\mathrm{Rh}(\mathrm{cod}) \mathrm{Cl}]_{2} /} \\
\mathrm{P}(o-\text { tol })_{3}{ }^{d}\end{array}$ & 194 & $24: 76$ & 89 \\
\hline 6 & $\begin{array}{l}{[\mathrm{Rh}(\text { cod }) \mathrm{Cl}]_{2} /} \\
\mathrm{P}(\text { mesityl })_{3}{ }^{d}\end{array}$ & 212 & $38: 62$ & 90 \\
\hline
\end{tabular}

aa $(0.44 \mathrm{mmol}), 4(0.88 \mathrm{mmol})$ was stirred in $\mathrm{CH}_{2} \mathrm{Cl}_{2}(4 \mathrm{~mL})$ in the presence of catalyst $(0.4 \mathrm{~mol} \%$ of metal) at $\mathrm{rt}$ for $6 \mathrm{~h}$ under argon. ${ }^{b}$ Determined by ${ }^{1} \mathrm{H}$ NMR spectroscopy of the crude reaction mixture. ${ }^{c} 1.7$ and $4.8 \mathrm{~mol} \%$ of TPPTS and $\mathrm{NBu}_{4} \mathrm{Cl}$ respectively used. ${ }^{d} 1.7 \mathrm{~mol} \%$ of phosphine was used. 
Turnover frequency measurement. It was determined following the representative procedure described above using ethyl 4-acetylbenzoate $\mathbf{3 f}$ as starting ketone and catalyst $\mathbf{1}$. But after $60 \mathrm{~min}$ stirring at $\mathrm{rt}$, the reaction mixture was filtered-off through celite, and concentrated under reduced pressure. A $59 \%$ conversion and ratios 5f:6f $=97: 3$ were measured by ${ }^{1} \mathrm{H}$ NMR spectroscopy of the crude reaction mixture, leading to a TOF $=124 \mathrm{~h}^{-1}$.

Recycling experiments. They were performed in a $12 \mathrm{~mL}-$ Supelco syringe on Quest ${ }^{\circledR}$ Parallel Synthesizer (Available from Argonaut Technologies (www.argotech.com) following the representative procedure described above using ethyl 4-acetylbenzoate $\mathbf{3 f}$ as starting ketone and catalyst $\mathbf{1}$. After completion of the reaction, the reaction mixture was then drained, the filtrate was concentrated under reduced pressure. \% conversion and ratio 5f:6f was measured by ${ }^{1} \mathrm{H}$ NMR spectroscopy of the crude reaction mixture. The catalyst was washed with dry $\mathrm{CH}_{2} \mathrm{Cl}_{2}(2 \times 4 \mathrm{~mL})$, dried by draining the $10 \mathrm{~mL}$ reactor with air. The catalytic beads kept in the reaction vessel were directly used for the second cycle using the same procedure described above. This process allowed us to keep constant the quantity of catalyst engaged for the dehydrogenative silylation reaction. At the end of the reaction, lower catalytic activity and selectivity were reached, with respectively $68 \%$ conversion and $\mathbf{5 f : 6 f}=95: 5$.

ICP-AES analysis of the supernatant solution at the end of the first indicated the level of palladium to be respectively $2 \mathrm{ppm}$, corresponding to an original metal loss of less than $0.4 \%$.

Sample preparation for TEM studies. After homogenization, a $5 \mu \mathrm{L}$ drop of a suspension of poly-ionic gel-soaked Rh $\mathbf{1}$ is placed onto a carbon-coated 400 mesh copper grid. After 1 minute adsorption, the excess of liquid is removed with a piece of filter paper and the grid is air dried. Transmission electron microscopy (TEM) observation was performed with a Philips CM-12 microscope operating at $120 \mathrm{kV}$. The images are taken with a SIS Megaview III camera at a nominal magnification of 66000 times. 


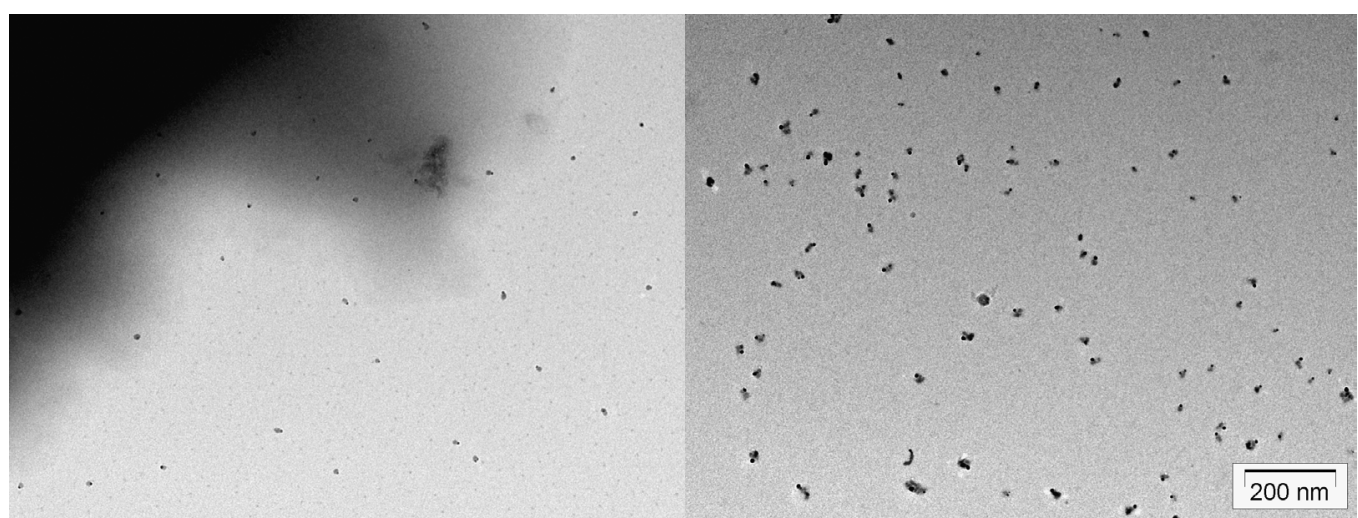

(a) Before reaction

(b) After reaction

Figure S1. Representative TEM images of poly-ionic gel-soaked Rh 1.

TEM analysis of $\mathbf{1}$ indicated the presence of Rh particles. (a) The size of the particles is ranging from 4 to $6 \mathrm{~nm}$, with a mean value of $5 \pm 1 \mathrm{~nm}$. (b) A larger amount of particles was detected. The size of the particles is ranging from 10 to $15 \mathrm{~nm}$, with a mean value of $12 \pm 1$ $\mathrm{nm}$.

\section{Heterogeneity Tests}

\section{Filtration tests. Rhodium Leaching.}

(a) Experiment 1. ${ }^{11}$ To a suspension of Rh catalyst 1 (13.1 mg, $2.09 \mu \mathrm{mol}, 0.16 \mathrm{mmol} \mathrm{Rh} / \mathrm{g}$ ) in dry $\mathrm{CH}_{2} \mathrm{Cl}_{2}(1 \mathrm{~mL})$ was added a solution of acetophenone $\mathbf{3 f}(84.5 \mathrm{mg}, 0.44 \mathrm{mmol}, 1$ equiv in $1 \mathrm{~mL}$ of dry $\mathrm{CH}_{2} \mathrm{Cl}_{2}$ ). The resulting mixture was stirred at room temperature for $10 \mathrm{~min}$, and then cooled to $0{ }^{\circ} \mathrm{C}$. A solution of diphenylsilane $4(163 \mu \mathrm{L}, 0.88 \mathrm{mmol}, 2$ equiv in $2 \mathrm{~mL}$ of dry $\mathrm{CH}_{2} \mathrm{Cl}_{2}$ ) was added dropwise. The stirred reaction solution was maintained for $1 \mathrm{~h}$ at $0^{\circ} \mathrm{C}$ and then was allowed to stir at room temperature for $30 \mathrm{~min}$. At this stage $(29 \%$ conversion and 5f:6f = 97:3), the catalyst was filtered off and the experiment was continued with the filtrate for another $5 \mathrm{~h} 30$. The reaction mixture was filtered off through celite, and concentrated under reduced pressure. A $31 \%$ conversion and a ratio 5f:6f $=97: 3$ were measured by ${ }^{1} \mathrm{H}$ NMR spectroscopy of the crude reaction mixture. To ensure that the catalyst is not killed during filtration, the same experiment was carried out. But after $30 \mathrm{~min}$ stirring at room temperature (27\% conversion and $\mathbf{5 f : 6 f}=98: 2)$, only supernatant was engaged without filtration for a further 5 h30 reaction. A $29 \%$ conversion was measured with a $\mathbf{5 f}: \mathbf{6 f}=99: 1$. 
(b) Experiment 2. To a suspension of Rh catalyst 1 (13.1 mg, $2.09 \mu \mathrm{mol}, 0.16 \mathrm{mmol} \mathrm{Rh} / \mathrm{g})$ in dry $\mathrm{CH}_{2} \mathrm{Cl}_{2}(1 \mathrm{~mL})$ was added a solution of acetophenone $\mathbf{3 f}(84.5 \mathrm{mg}, 0.44 \mathrm{mmol}, 1$ equiv in $1 \mathrm{~mL}$ of dry $\mathrm{CH}_{2} \mathrm{Cl}_{2}$ ). The resulting mixture was stirred at room temperature for $10 \mathrm{~min}$, and then cooled to $0{ }^{\circ} \mathrm{C}$. A solution of diphenylsilane $4(163 \mu \mathrm{L}, 0.88 \mathrm{mmol}, 2$ equiv in $2 \mathrm{~mL}$ of dry $\mathrm{CH}_{2} \mathrm{Cl}_{2}$ ) was added dropwise. The stirred reaction solution was maintained for $1 \mathrm{~h}$ at $0^{\circ} \mathrm{C}$ and then was allowed to stir at room temperature for $6 \mathrm{~h}$. The catalyst was filtered off and a $98 \%$ conversion was measured by ${ }^{1} \mathrm{H}$ NMR spectroscopy of the crude reaction mixture. Rh leaching in the solution was measured by ICP-AES to be $2 \mathrm{ppm}$, corresponding to an original metal loss of $0.4 \%$. The flask received an additional charge of acetophenone 3a (53 $\mathrm{mg}, 0.44$ mmol, 1 equiv in $1 \mathrm{~mL}$ of dry $\left.\mathrm{CH}_{2} \mathrm{Cl}_{2}\right)$ and a solution of diphenylsilane 4 (163 $\mu \mathrm{L}, 0.88$ mmol, 2 equiv in $2 \mathrm{~mL}$ of dry $\mathrm{CH}_{2} \mathrm{Cl}_{2}$ ), and the experiment was continued for another $6 \mathrm{~h}$ at room temperature. The filtrate was concentrated under reduced pressure. Conversion was determined by ${ }^{1} \mathrm{H}$ NMR spectroscopy of the crude reaction mixture. No reaction was observed.

\section{Three phase Test. $^{12}$}

This heterogeneity test was not performed due to the instability of the diphenylsilyl enols and their incompatibly with the cleavage conditions.

General Procedure for the One-Pot Mukaiyama Aldol Reaction. ${ }^{13}$ A solution of the reaction mixture containing DPS enol $5 \mathbf{a}(6 \mathrm{~mL}, 0.66 \mathrm{mmol}, 1.5$ equiv) was added dropwise into a mixture of $44.7 \mu \mathrm{L}$ (0.44 mmol, 1.0 equiv) of benzaldehyde 7 and $55.7 \mu \mathrm{L}$ (0.44 mmol, 1.0 equiv) of $\mathrm{BF}_{3} \cdot \mathrm{Et}_{2} \mathrm{O}$ in dry $\mathrm{CH}_{2} \mathrm{Cl}_{2}(5 \mathrm{~mL})$ under an argon atmosphere at $-78^{\circ} \mathrm{C}$, and the reaction mixture was stirred for $1 \mathrm{~h}$. After hydrolysis at that temperature, the resulting organic layer was extracted with ether, and the extract was washed with water and dried over anhydrous $\mathrm{Na}_{2} \mathrm{SO}_{4}$. The mixture was condensed under reduced pressure, and the crude product was chromatographed on silica gel. Elution with cyclohexane, then cyclohexaneethyl acetate (5:1) mixture gave $84.5 \mathrm{mg}(85 \%)$ of $\mathbf{8}$ as a colorless oil.

1,3-Diphenyl-3-hydroxy-1-propanone 8. ${ }^{14}$ (Silica gel, cyclohexane:ethyl acetate $=5: 1$ ). 85\% yield. Colorless oil. $R_{\mathrm{f}}=0.37\left(\mathrm{SiO}_{2}\right.$, cyclohexane:ethyl acetate $\left.=8: 2\right) .{ }^{1} \mathrm{H}$ NMR $(200 \mathrm{MHz}$, $\left.\mathrm{CDCl}_{3}\right): 3.32(\mathrm{dd}, J=3.9,17.6 \mathrm{~Hz}, 1 \mathrm{H}), 3.44(\mathrm{dd}, J=8.1,17.6 \mathrm{~Hz}, 1 \mathrm{H}), 3.89$ (s, $\left.1 \mathrm{H}\right), 5.38$ $(\mathrm{dd}, J=3.9,8.1 \mathrm{~Hz}, 1 \mathrm{H}), 6.54-6.57(\mathrm{~m}, 2 \mathrm{H}), 7.28-7.65(\mathrm{~m}, 8 \mathrm{H}), 7.95-7.98(\mathrm{~m}, 2 \mathrm{H}) .{ }^{13} \mathrm{C} \mathrm{NMR}$ 
$\left(200 \mathrm{MHz}, \mathrm{CDCl}_{3}\right): \delta 47.9,70.4,126.3,128.0,128.6,128.9,129.1,134.0,137.1,143.7$, 200.3. GC-MS: [M+1] 227, [M-OH] 209. HRMS (EI) calcd for $\mathrm{C}_{15} \mathrm{H}_{14} \mathrm{O}_{2} 226.0994\left(\mathrm{M}^{+}\right)$, found 226.0988. IR (KRS 5): 1682 (vC=O), 3467 (vO-H).

4-Hydroxy-1,1-dimethoxy-4-(2'-pyridyl)butan-2-one 9. ${ }^{15}$ (Silica gel, cyclohexane:ethyl acetate $=2: 8) .75 \%$ yield. Yellow oil. $R_{\mathrm{f}}=0.13\left(\mathrm{SiO}_{2}\right.$, cyclohexane:ethyl acetate $\left.=5: 5\right) .{ }^{1} \mathrm{H}$ NMR (300 MHz, $\left.\mathrm{CDCl}_{3}\right): 3.08-3.11(\mathrm{~m}, 2 \mathrm{H}), 3.41(\mathrm{~s}, 3 \mathrm{H}), 3.42(\mathrm{~s}, 3 \mathrm{H}), 4.04$ (d, J = 5.6 Hz, $1 \mathrm{H}), 4.54(\mathrm{~s}, 1 \mathrm{H}), 5.22-5.28(\mathrm{~m}, 1 \mathrm{H}), 7.18-7.22(\mathrm{~m}, 1 \mathrm{H}), 7.43$ (d, $J=7.8 \mathrm{~Hz}, 1 \mathrm{H}), 7.70$ (td, $J=$ 1.9, $7.8 \mathrm{~Hz}, 1 \mathrm{H}), 8.52-8.55$ (m, 1H). ${ }^{13} \mathrm{C} \mathrm{NMR}\left(300 \mathrm{MHz}, \mathrm{CDCl}_{3}\right): \delta 45.6,54.5,69.3,103.9$, 120.3, 122.3, 136.7, 148.3, 161.3, 204.6. GC-MS: [M+1] 226. HRMS (EI) calcd for $\mathrm{C}_{11} \mathrm{H}_{15} \mathrm{NO}_{4} 225.1001\left(\mathrm{M}^{+}\right)$, found 225.1007. IR (KRS 5): 1074, 1438, 1594, $1733(v \mathrm{C}=\mathrm{O})$, 2940, 3449 (vO-H).

Ethyl 5-(3',4',5'-trimethoxyphenyl)-5-hydroxy-2-methyl-3-oxopentanoate 10. (Silica gel, cyclohexane:ethyl acetate $=5: 5) .70 \%$ yield as inseparable mixture of syn and anti diastereomers in 1:1. Colorless oil. $R_{\mathrm{f}}=0.40\left(\mathrm{SiO}_{2}\right.$, cyclohexane:ethyl acetate $\left.=5: 5\right) .{ }^{1} \mathrm{H}$ NMR (300 MHz, $\left.\mathrm{CDCl}_{3}\right): 1.17-1.24(\mathrm{~m}, 3 \mathrm{H}), 1.29$ (d, $\left.J=7.1 \mathrm{~Hz}, 3 \mathrm{H}\right), 2.77-3.02(\mathrm{~m}, 2 \mathrm{H})$, $3.27(\mathrm{~d}, J=3.1 \mathrm{~Hz}, 1 \mathrm{H}), 3.44-3.55(\mathrm{~m}, 1 \mathrm{H}), 3.76(\mathrm{~s}, 3 \mathrm{H}), 3.80(\mathrm{~s}, 6 \mathrm{H}), 4.09-4.17(\mathrm{~m}, 2 \mathrm{H})$, 5.04-5.07 (m, 1H), $6.52(\mathrm{~s}, 2 \mathrm{H}) .{ }^{13} \mathrm{C} \mathrm{NMR}\left(300 \mathrm{MHz}, \mathrm{CDCl}_{3}\right): \delta 12.5,14.0,50.1,50.4,53.4$, 56.0, 60.7, 61.5, 69.9, 70.1, 102.5, 137.2, 138.6, 153.3, 170.2, 205.8. GC-MS: [M+1] 341. HRMS (EI) calcd for $\mathrm{C}_{17} \mathrm{H}_{24} \mathrm{O}_{7} 340.1522\left(\mathrm{M}^{+}\right)$, found 340.1519. IR (KRS 5): 1127, 1235, 1459, 1593, $1712(v \mathrm{C}=\mathrm{O}), 1740(v \mathrm{C}=\mathrm{O}), 2941,3505(v \mathrm{O}-\mathrm{H})$.

\section{Procedure for the One-Pot Mannich Reaction.}

Benzylidene-(4-methoxy-phenyl)-amine. Prepared by a known method. ${ }^{16}$ The title compound was prepared by addition of $3.5 \mathrm{~g}$ ( $23.5 \mathrm{mmol}, 1$ equiv), and $50 \mathrm{~mL}$ of $\mathrm{H}_{2} \mathrm{O}$ to a $100 \mathrm{~mL}$ round bottom flask followed by $2.5 \mathrm{~g}$ (23.5 mmol, 1 equiv) of benzaldehyde. The mixture was stirred vigorously for $7 \mathrm{~h}$ open to ambient atmosphere then filtered, washing with $\mathrm{H}_{2} \mathrm{O}$. The product was purified by recristallization from hot EtOH to afford $4.2 \mathrm{~g}(86 \%)$ of the aldimine as brown flakes. Spectral data correspond to that previously reported. Mp 65-66 ${ }^{\circ} \mathrm{C} .{ }^{1} \mathrm{H}$ NMR $\left(300 \mathrm{MHz}, \mathrm{CDCl}_{3}\right): \delta 3.85(\mathrm{~s}, 1 \mathrm{H}), 6.93-6.96(\mathrm{~m}, 2 \mathrm{H})$, 7.24-7.27 (m, 2H), 7.47 $(\mathrm{t}, J=3.1 \mathrm{~Hz}, 3 \mathrm{H}), 7.89-7.92(\mathrm{~m}, 2 \mathrm{H}), 8.49(\mathrm{~s}, 1 \mathrm{H}) .{ }^{13} \mathrm{C} \mathrm{NMR}\left(300 \mathrm{MHz}, \mathrm{CDCl}_{3}\right): \delta 55.3$, 
114.2, 122.0, 128.4, 128.6, 130.9, 136.3, 144.7, 158.1, 158.3. GC-MS: [M+1] 212. HRMS (EI) calcd for $\mathrm{C}_{14} \mathrm{H}_{13} \mathrm{NO} 211.0997\left(\mathrm{M}^{+}\right)$, found 211.0990. IR (KRS 5): $1623(v \mathrm{C}=\mathrm{N})$.

1,3-Diphenyl-3-(N-4-methoxyphenyl)amino-1-propanone 11. Prepared by a known method. ${ }^{17}$ To $\mathrm{Yb}(\mathrm{OTf})_{3}\left(0.088 \mathrm{mmol}, 0.2\right.$ equiv) in $\mathrm{CH}_{2} \mathrm{Cl}_{2}(1 \mathrm{~mL})$ was added the previously prepared aldimine $\left(0.44 \mathrm{mmol}, 1\right.$ equiv) in $\mathrm{CH}_{2} \mathrm{Cl}_{2}(1 \mathrm{~mL})$. The mixture was cooled to $-23{ }^{\circ} \mathrm{C}$, and the reaction mixture containing DPS enol 5a $(6 \mathrm{~mL}, 0.66 \mathrm{mmol}, 1.5$ equiv) was added dropwise. The reaction mixture was stirred for $1 \mathrm{~h}$ at $-23{ }^{\circ} \mathrm{C}$. Water was added to quench the reaction, and the mixture was warmed to room temperature. After extraction of the aqueous layer with $\mathrm{CH}_{2} \mathrm{Cl}_{2}$, the resulting organic layer was washed with water and dried over anhydrous $\mathrm{Na}_{2} \mathrm{SO}_{4}$. The mixture was condensed under reduced pressure, and the crude product was chromatographed on silica gel. Elution with cyclohexane-ethyl acetate $(5: 1)$ mixture gave $131.1 \mathrm{mg}$ (90\%) of $\mathbf{1 1}$ as brownish wet-solid. Spectral data correspond to that

previously reported. ${ }^{18} \mathrm{Mp} 140-141{ }^{\circ} \mathrm{C} .{ }^{1} \mathrm{H}$ NMR (300 MHz, $\mathrm{CDCl}_{3}$ ): 3.42 (dd, $J=7.1,16.2$ $\mathrm{Hz}, 1 \mathrm{H}), 3.51$ (dd, $J=5.2,16.2 \mathrm{~Hz}, 1 \mathrm{H}), 3.71$ (s, 3H), 4.95 (dd, $J=5.2,7.1 \mathrm{~Hz}, 1 \mathrm{H}), 6.54-$ $6.57(\mathrm{~m}, 2 \mathrm{H}), 6.69-6.72(\mathrm{~m}, 2 \mathrm{H}), 7.25-7.58(\mathrm{~m}, 8 \mathrm{H}), 7.92-7.95(\mathrm{~m}, 2 \mathrm{H}) .{ }^{13} \mathrm{C}$ NMR $(200 \mathrm{MHz}$, $\left.\mathrm{CDCl}_{3}\right): \delta 46.8,56.1,56.1,115.1,115.8,126.8,127.7,128.6,129.0,129.2,133.7,137.2$, 141.5, 143.6, 152.8, 198.7. GC-MS: 123, 209, 212. HRMS (EI) calcd for $\mathrm{C}_{22} \mathrm{H}_{21} \mathrm{NO}_{2}$ 331.1572 $\left(\mathrm{M}^{+}\right)$, found 331.1569. IR (KRS 5): $1683(\mathrm{vC}=\mathrm{O}), 3375(\mathrm{vN}-\mathrm{H})$.

\section{References}

(1) Thiot, C.; Schmutz, M.; Wagner, A.; Mioskowski, C. Angew. Chem. Int. Ed. 2006, 45, 2868.

(2) Yao, S.; Meng, J-C.; Siuzdak, G.; Finn, M.G. J. Org. Chem. 2003, 68, 2540.

(3) Brunner, H. ; Obermann, U. Chem. Ber. 1989, 122, 499.

(4) Ojima, I. ; Kogure, T. Organometallics 1982, 1, 1390.

(5) Zhang, L; Kozmin, S-A. J. Am. Chem. Soc. 2004, 126, 10204.

(6) Spears, G. W.; Caufield, C. E.; Still, W. C. J. Org. Chem. 1987, 52, 1226.

(7) Aggarwal, V. K.; Sheldon, C. G.; Macdonald, G. J.; Martin, W. P. J. Am. Chem. Soc. 2002, 124, 10300.

(8) Ahmad, S.; Khan, M.A; Iqbal, J, Synthetic Communications 1988, 18, 1679.

(9) Poirier, J.M.; Hennequin, J.; Fomami, M., Bull. Soc. Chim. Fr. 1986, 3, 436.

(10) Bach, T.; Joedicke, K. Chem. Ber. 1993, 126, 2457.

(11) Sheldon, R. A.; Wallau, M.; Arends, I. W. C. E.; Schuchardt, U. Acc. Chem. Res. 1998, $31,485$. 
(12) (a) Rebek, J.; Gavina, F. J. Am. Chem. Soc. 1974, 96, 7112. (b) Rebek, J.; Brown, D.; Zimmerman, S. J. Am. Chem. Soc. 1975, 97, 454. (c) Davies, I. W.; Matty, L.; Hughes, D. L.; Reider, P. J. J. Am. Chem. Soc. 2001, 123, 10139. (d) Crudden, C. M.; Sateesh, M.; Lewis, R. J. Am. Chem. Soc. 2005, 127, 10045.

(13) Mukaiyama, T. ; Banno, K. ; Narasaka, K. J. Am. Chem. Soc. 1974, 96, 7503.

(14) Le Roux, C. ; Gaspard-I, H. ; Dubac, J. J. Org. Chem. 1993, 58, 1835.

(15) Chimni, S. S.; Mahajan, D. Tetrahedron 2005, 61, 5019.

(16) Bowman, R.K.; Johnson, J.S. J. Org. Chem. 2004, 69, 8537.

(17) Kobayashi, S. ; Nagayama, S. J. Org. Chem. 1997, 62, 232.

(18) Loh, T-P.; Liung, S.; Tan, K-L.; Wie, L-L. Tetrahedron 2000, 56, 3227. 


\section{${ }^{31} \mathrm{P}$ NMR of the sulfonated phosphine poly-ionic gel}
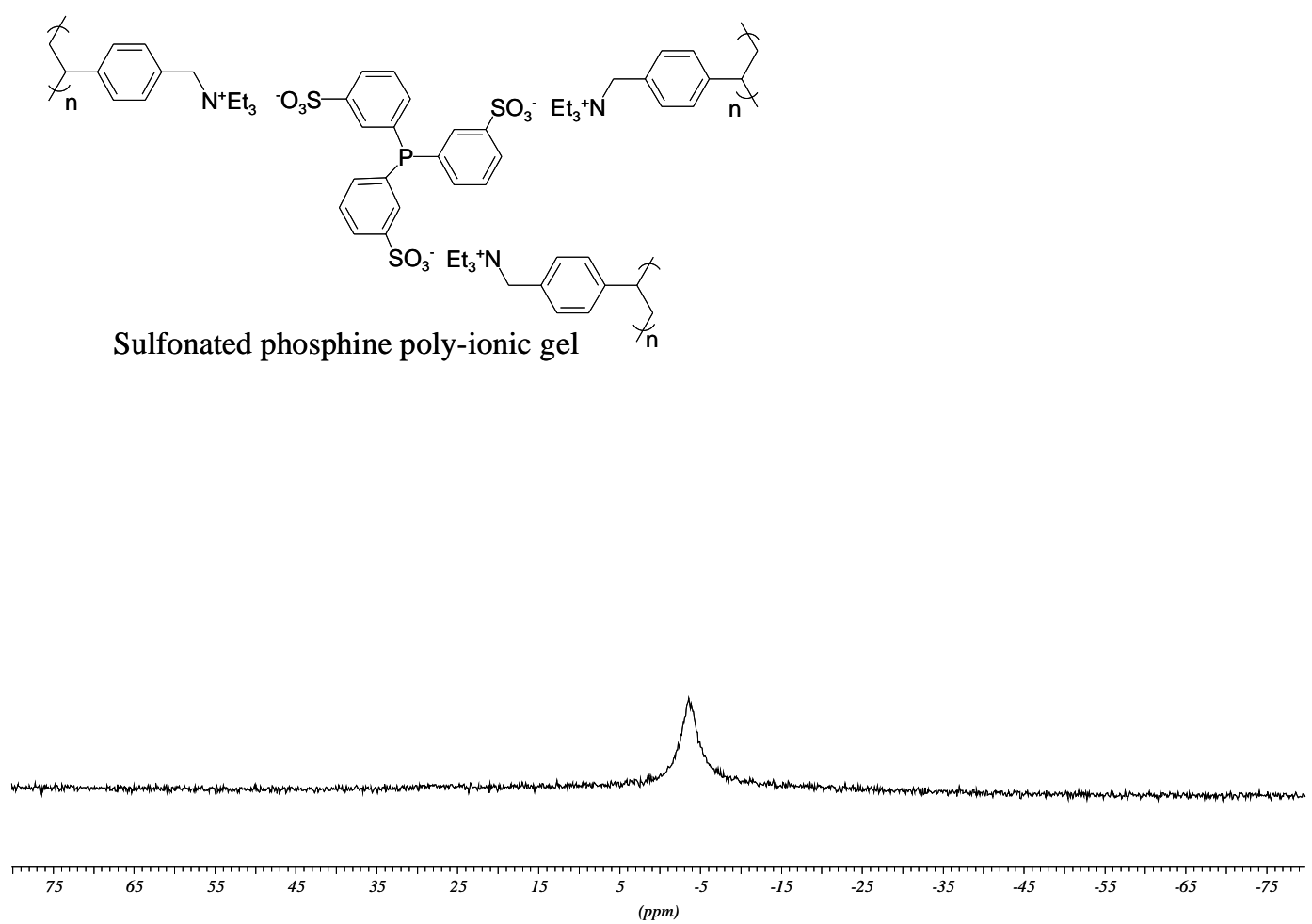

\section{${ }^{31} \mathrm{P}$ NMR of 1}
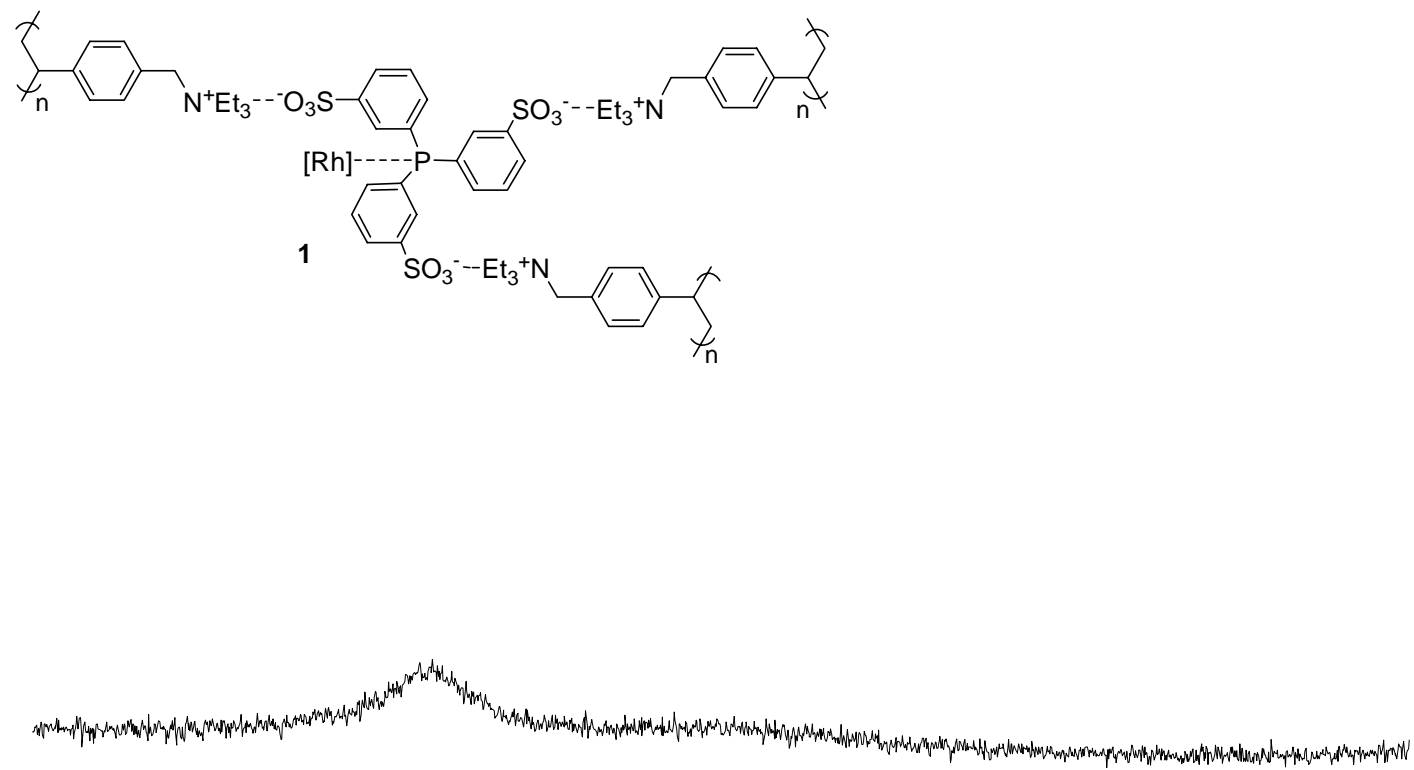

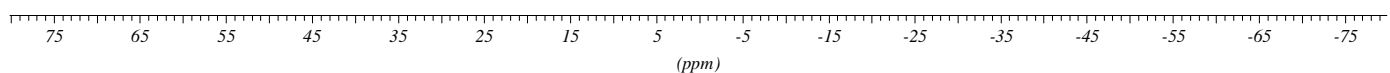


${ }^{31} \mathrm{P}$ NMR of 2
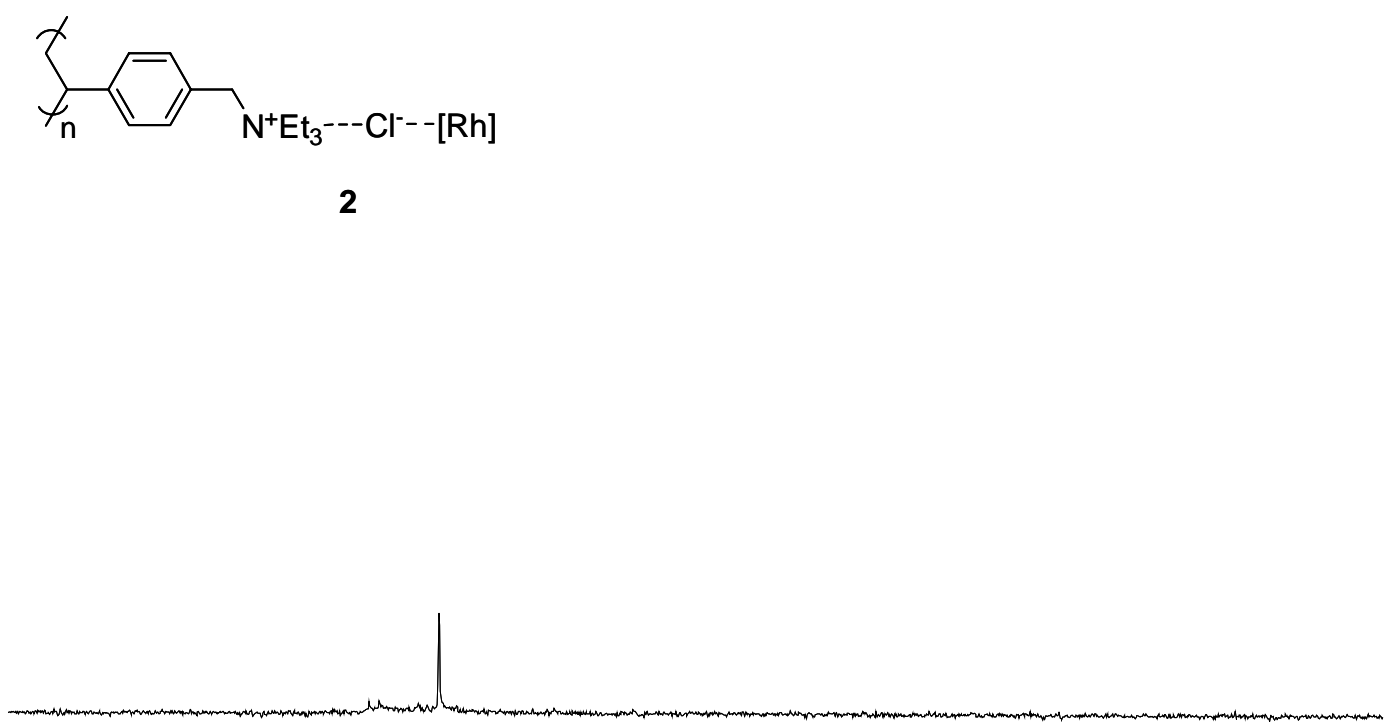

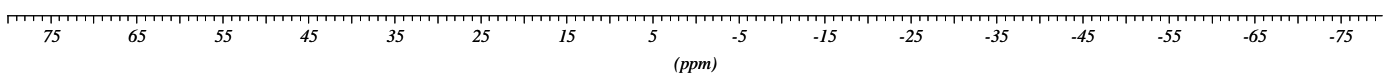




\section{${ }^{1} \mathrm{H}$ NMR of crude $5 f$}<smiles>C=C(O[Ga](c1ccccc1)c1ccccc1)c1ccc(C(=O)OCC)cc1</smiles>
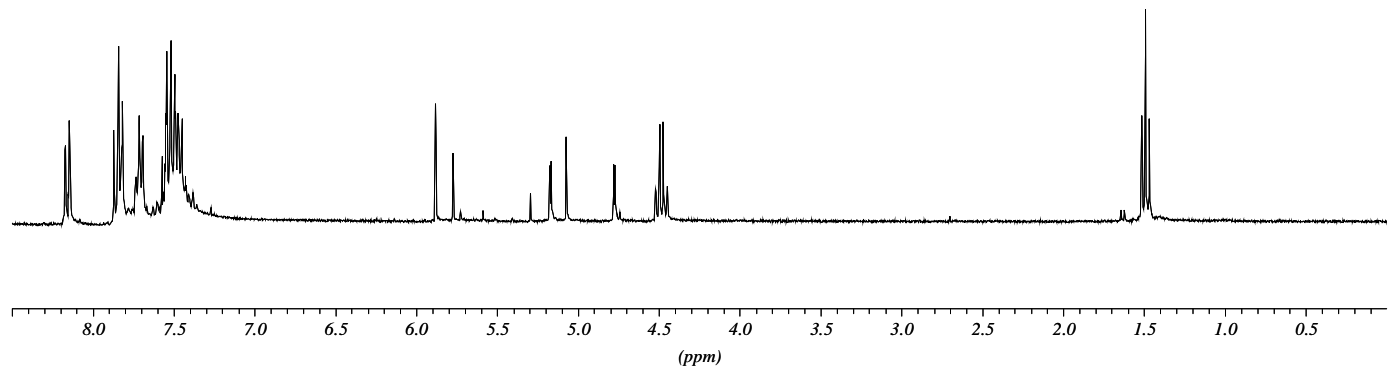

\section{${ }^{1} \mathrm{H}$ NMR of crude $3 f$}<smiles>CCOC(=O)c1ccc(C(C)=O)cc1</smiles>

3f: : reduced ketone $=96: 4$ after hydrolysis $1 \% \mathrm{TsOH} / \mathrm{MeOH}$
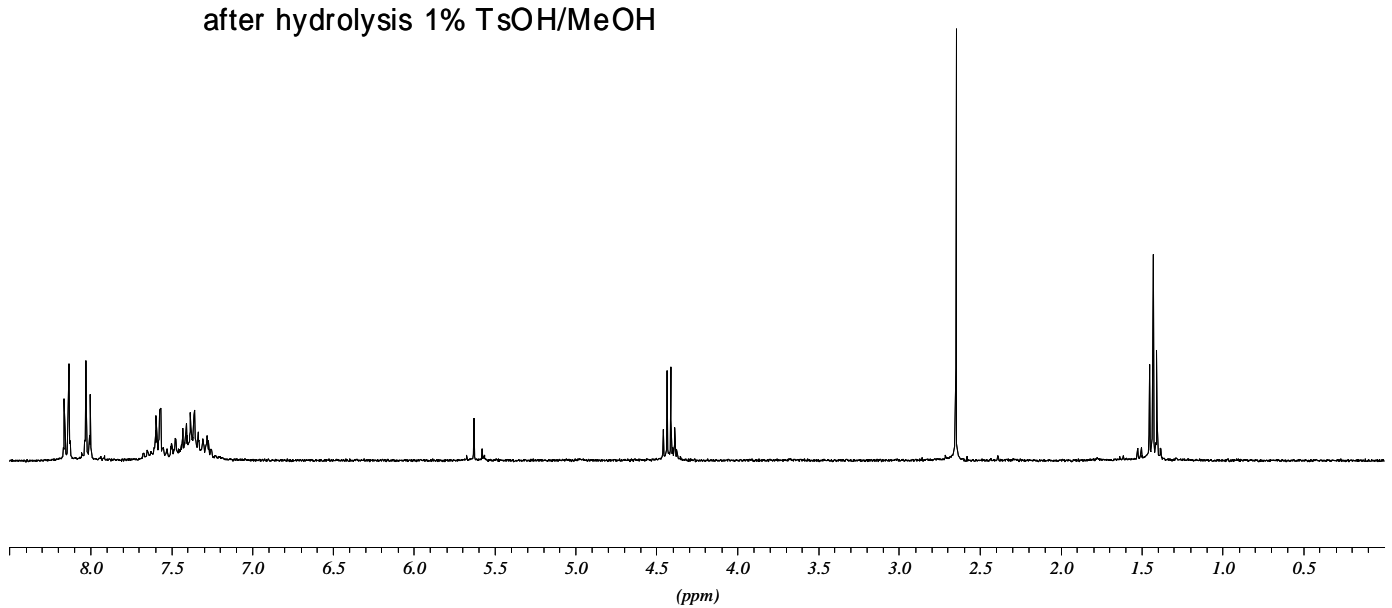


\section{${ }^{1} \mathrm{H}$ NMR of crude $5 \mathbf{r}$}
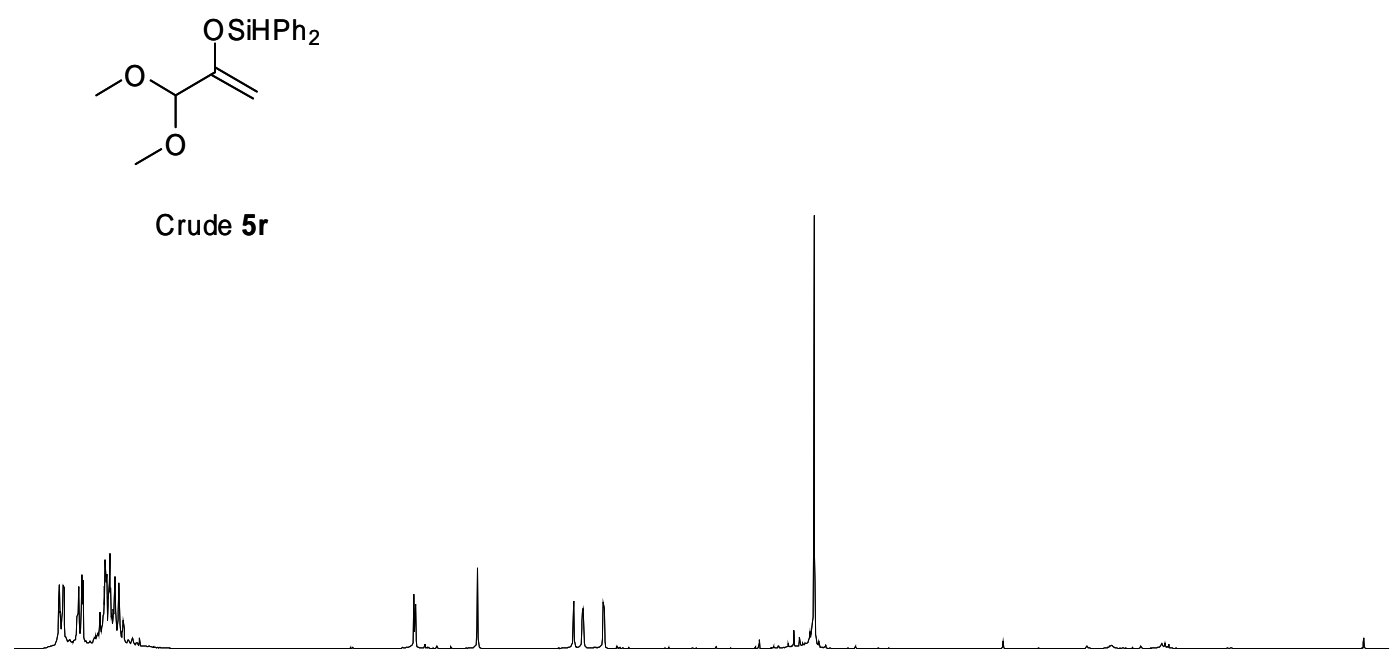

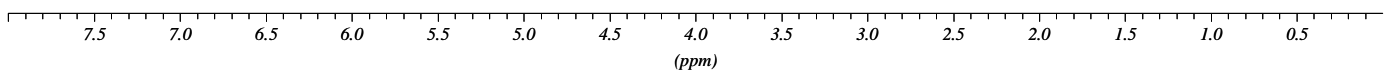

\section{${ }^{1} \mathrm{H}$ NMR of crude $3 \mathbf{r}$}

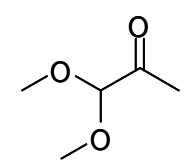

3r after hydrolysis $1 \% \mathrm{TsOH} / \mathrm{MeOH}$

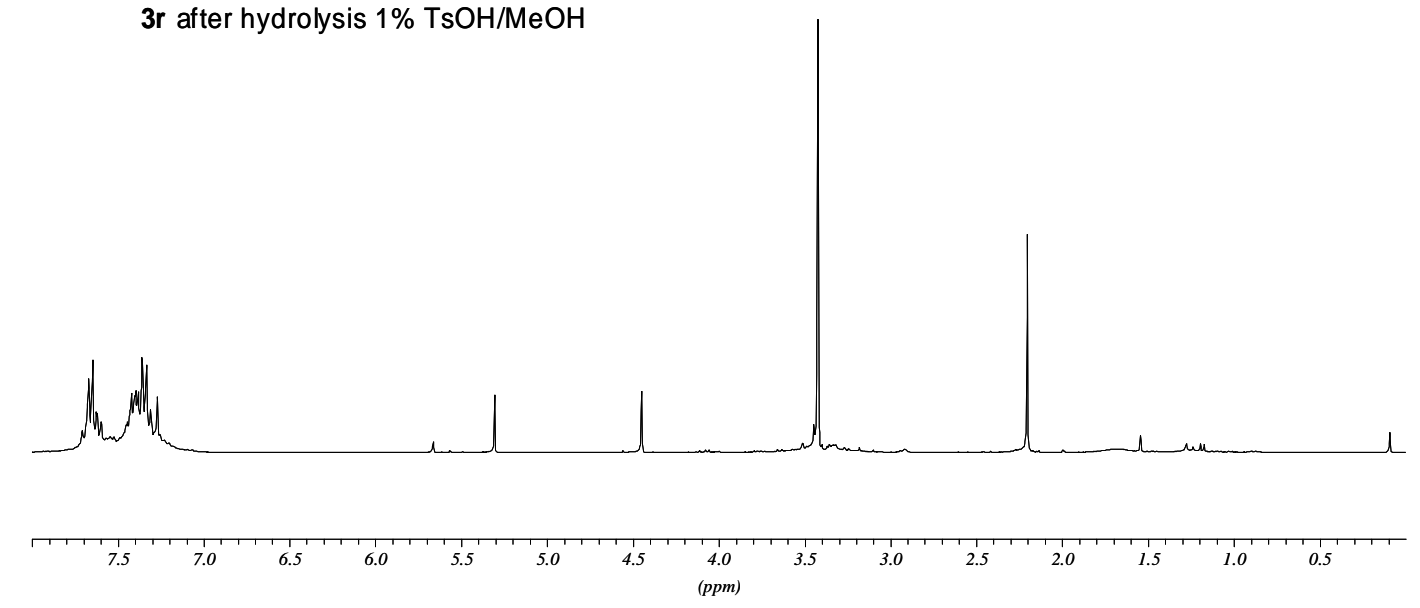


Gaschromatograph:

Software:

Column:

Carrier:

Injector:

Detector:

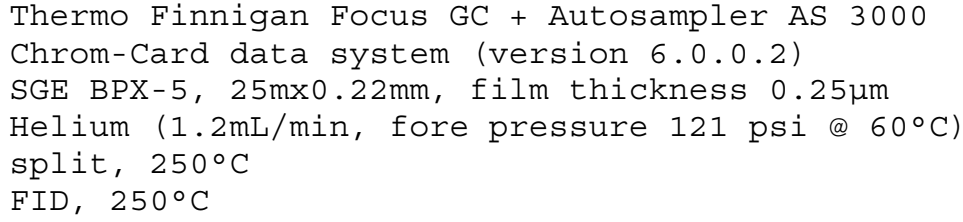

GC of crude $5 r . R_{t} \sim 7.292 \min \left(\right.$ silylated by-product $: R_{t} \sim 8.857$ min)

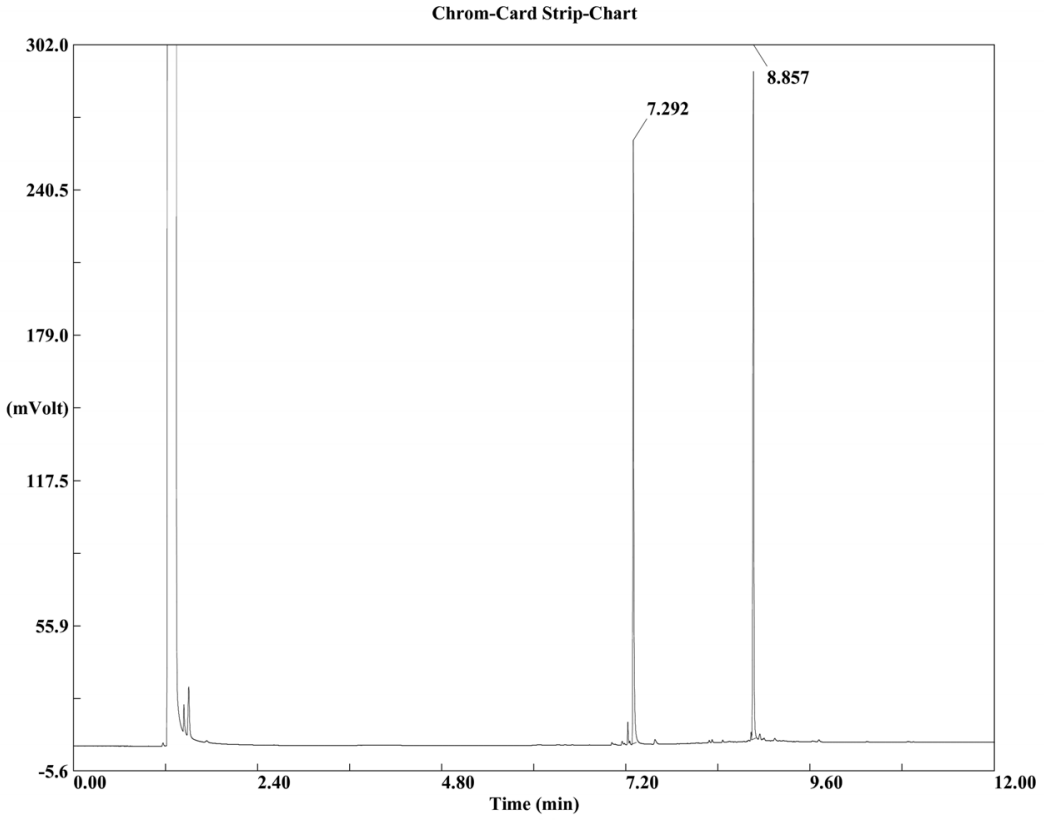

GC after hydrolysis of $5 r .3 r: R_{t} \sim 2.672$ min (silylated by- product $: R_{t} \sim 8.857$ min)

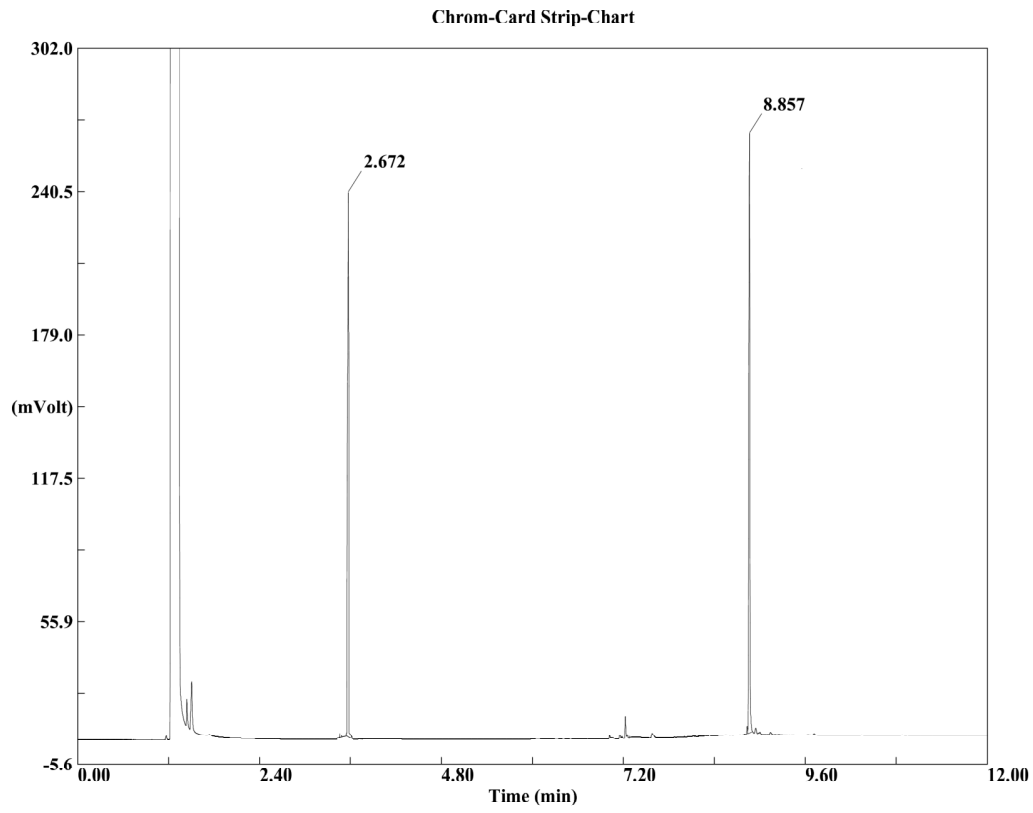


${ }^{1}$ H NMR of crude $5 t$ (18\% of $3 t$ remaining)

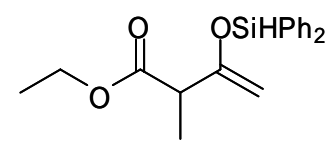

Crude 5t
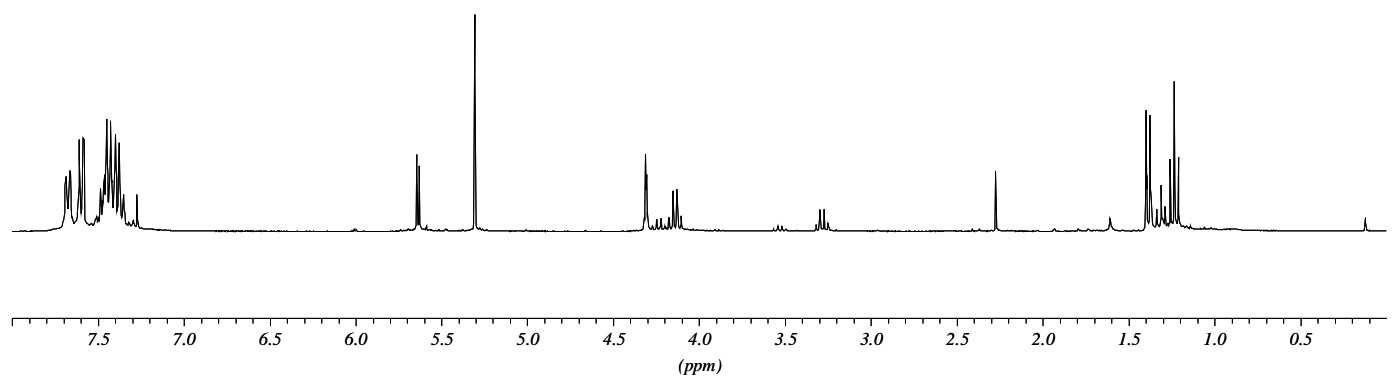

\section{${ }^{1}$ H NMR of crude $3 t$}

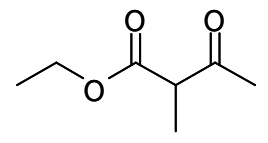

3t after hydrolysis $1 \% \mathrm{TsOH} / \mathrm{MeOH}$
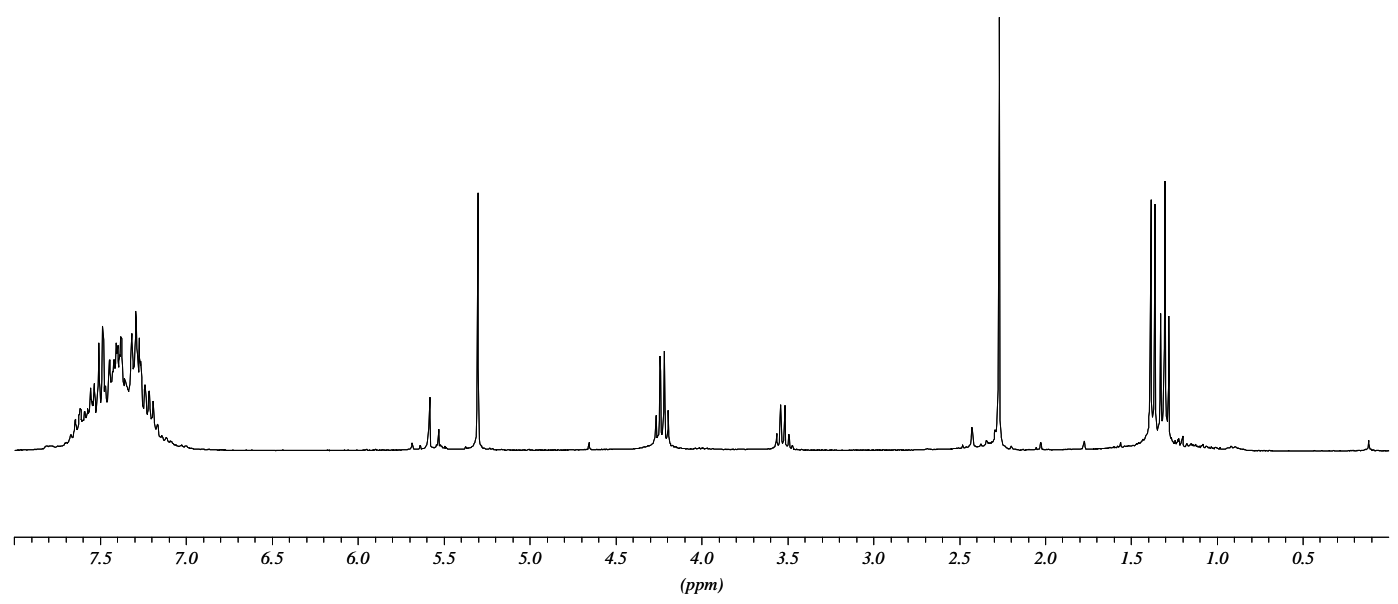
GC of crude $5 t . R_{t} \sim 7.617$ min (silylated by-product $: R_{t} \sim 8.858$ min)

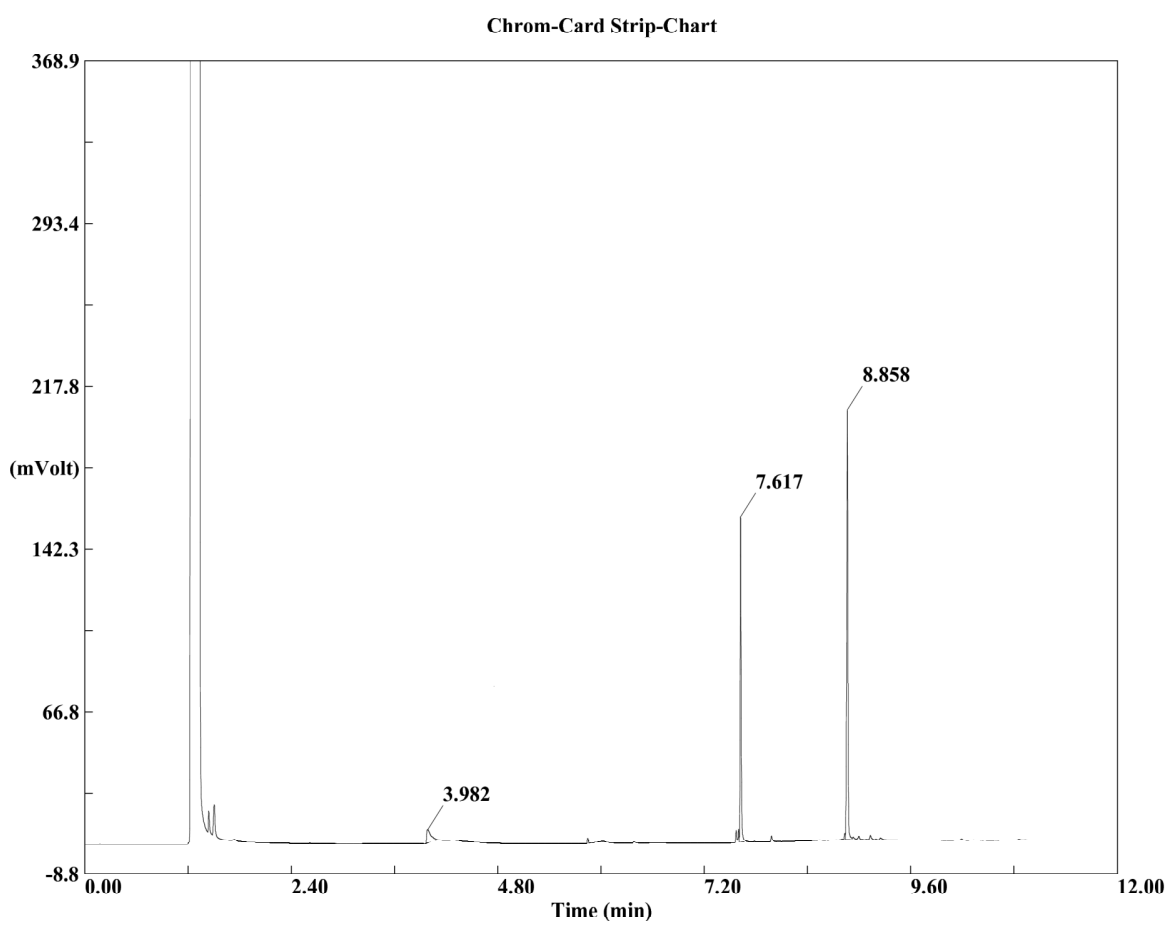

GC after hydrolysis of 5t. $3 t: R_{t} \sim 3.984$ min (silylated by-product $: R_{t} \sim 8.857$ min)

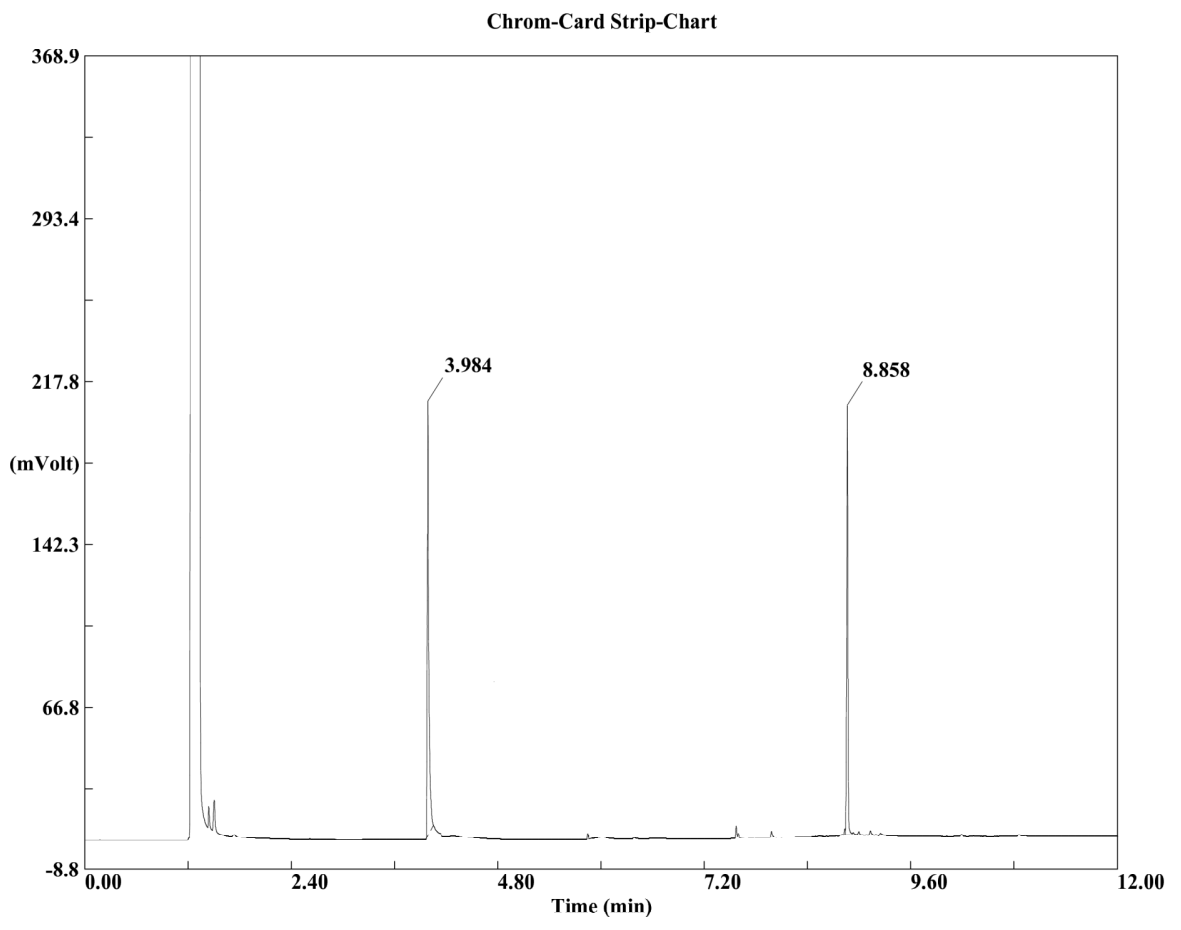




\section{${ }^{1} \mathrm{H}$ NMR of 8}<smiles>O=C(CC(O)c1ccccc1)c1ccccc1</smiles>
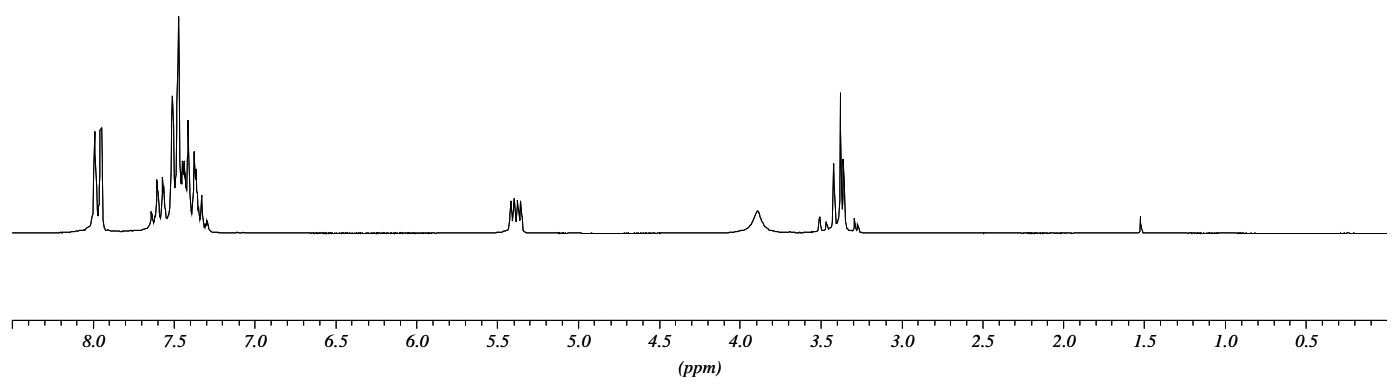

\section{${ }^{13} \mathrm{C}$ NMR of 8}<smiles>O=C(CC(O)c1ccccc1)c1ccccc1</smiles>
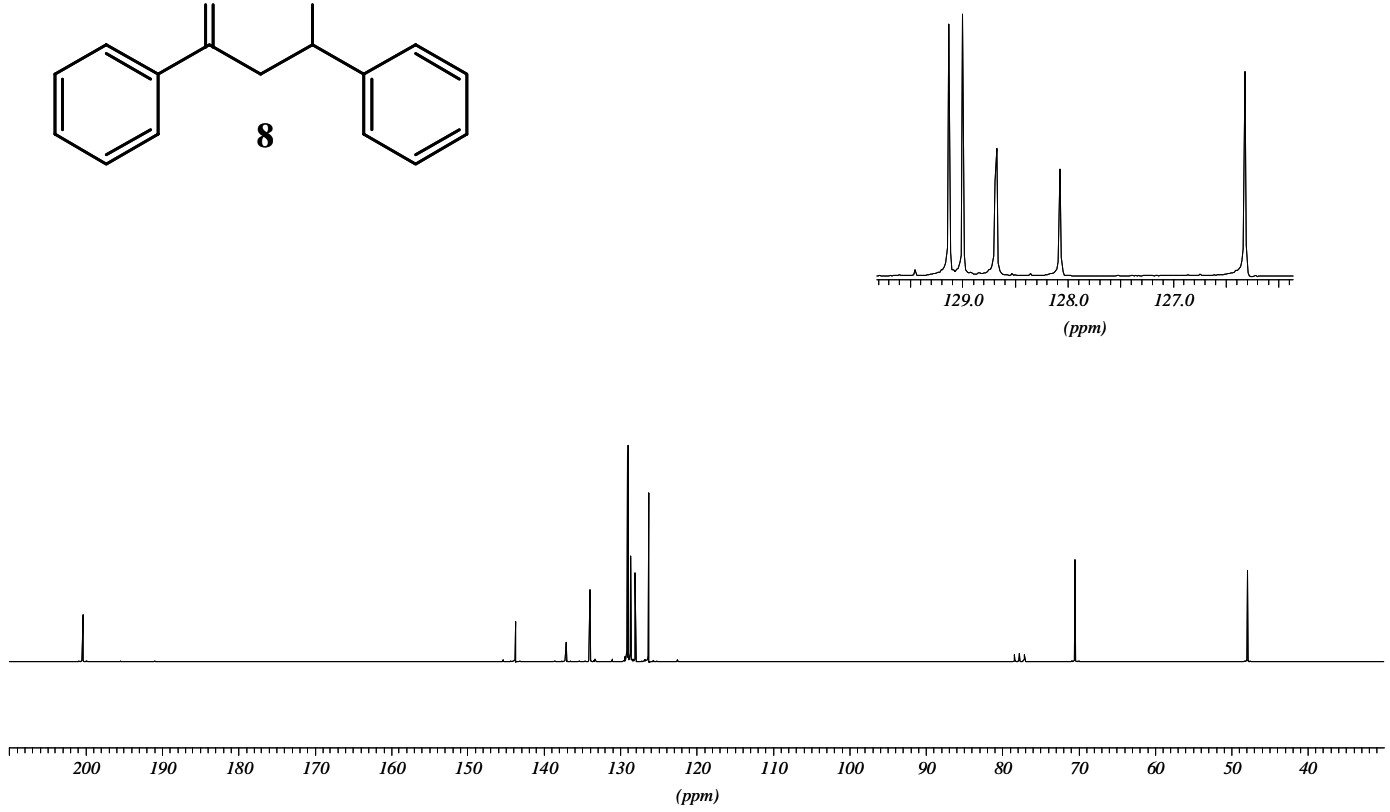


\section{${ }^{1} \mathrm{H}$ NMR of 9}
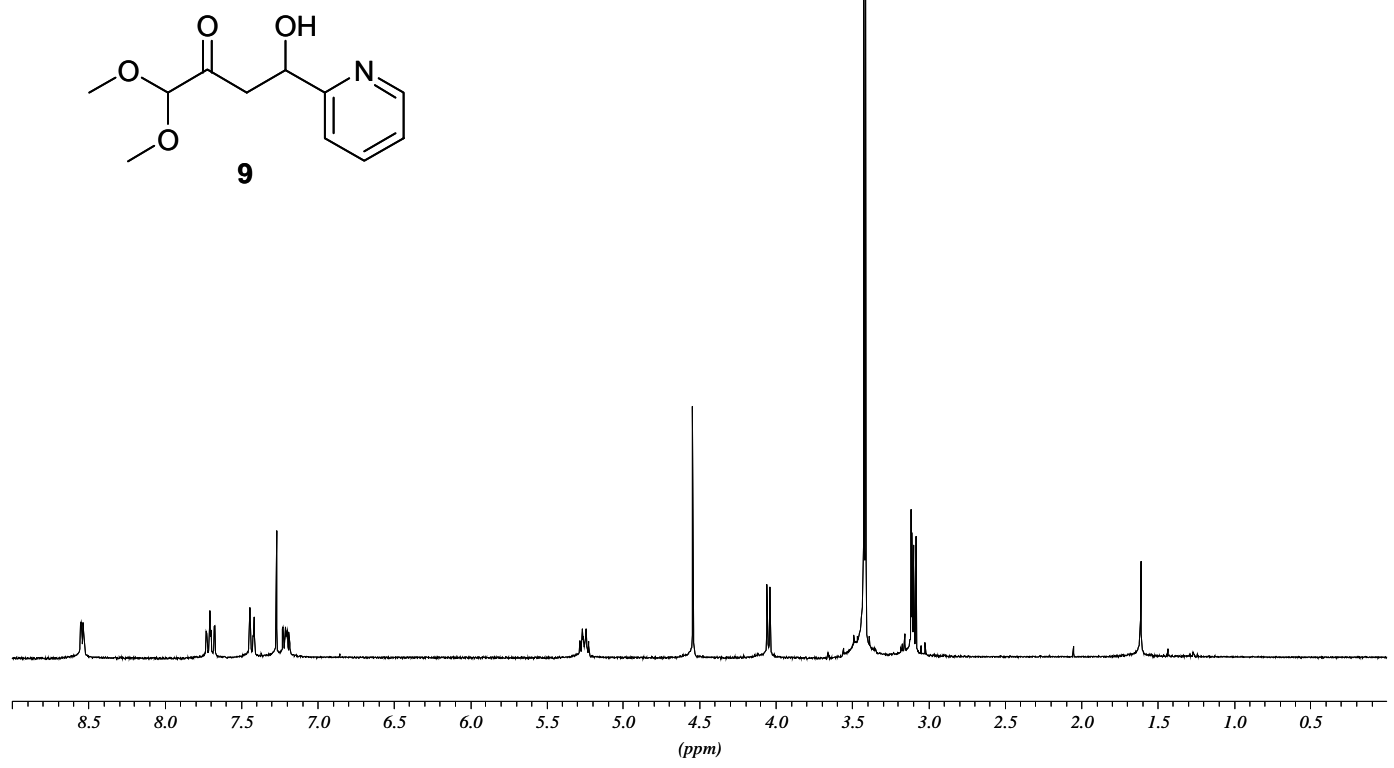

${ }^{13}$ C NMR of 9
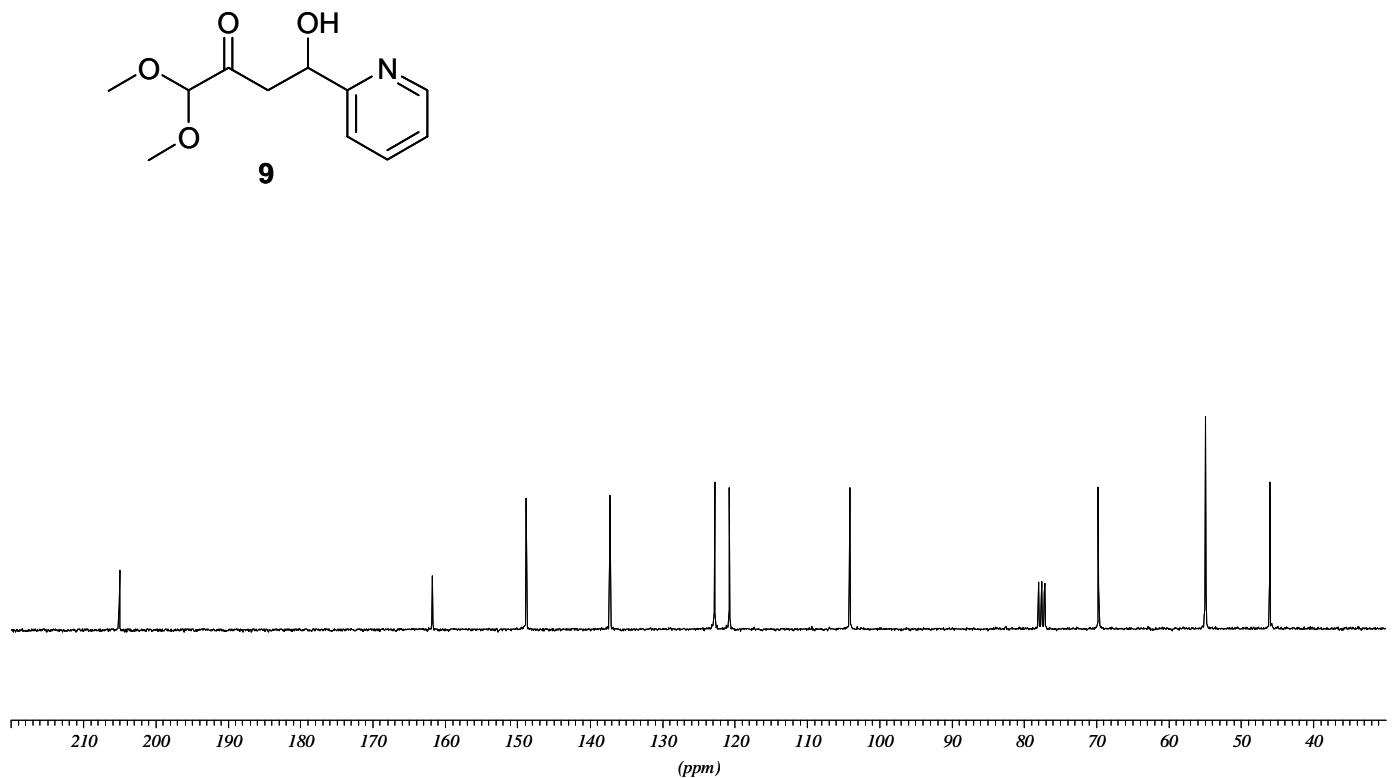


\section{${ }^{1} \mathrm{H}$ NMR of 10}
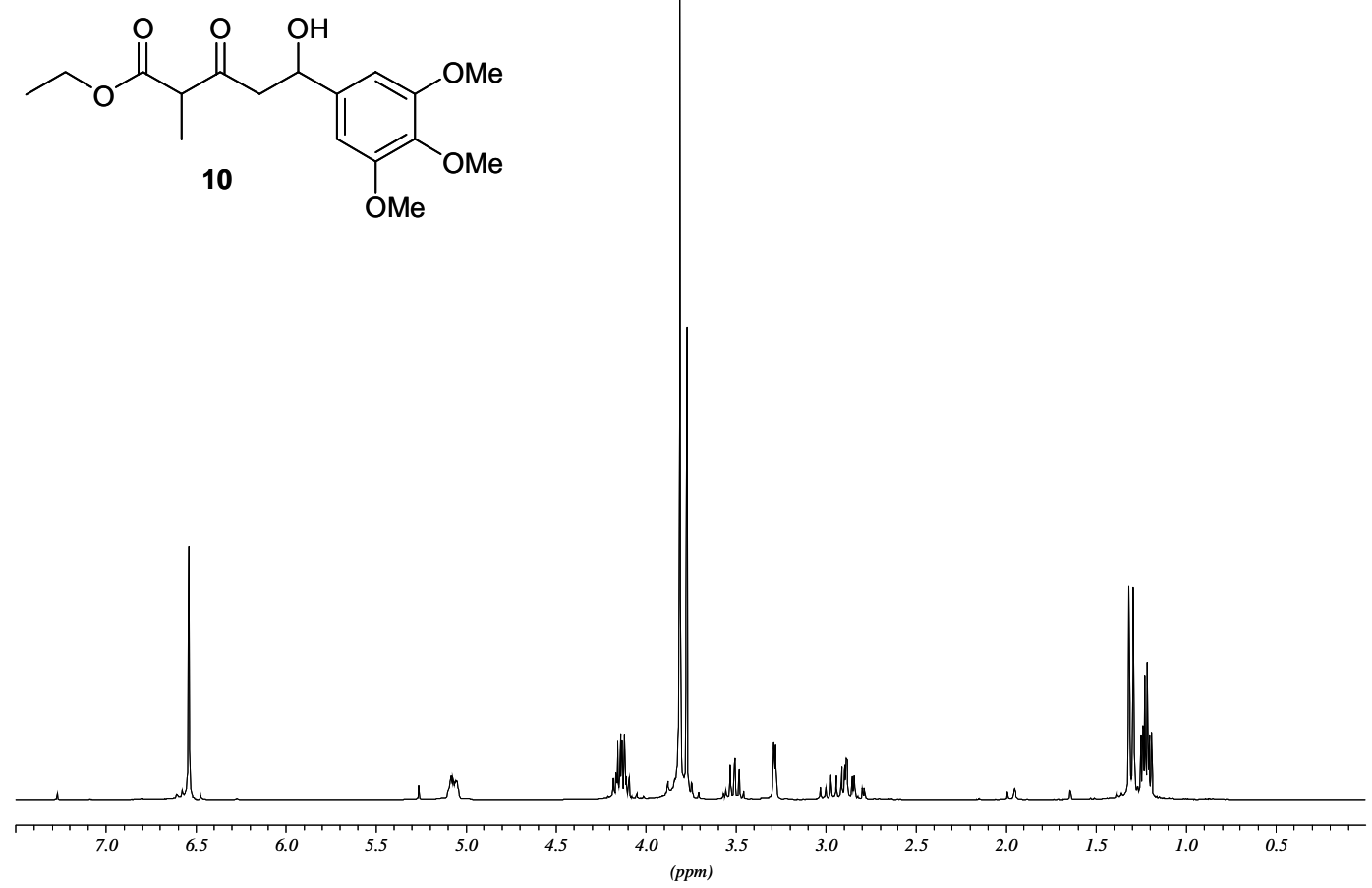

\section{${ }^{13} \mathrm{C}$ NMR of 10}
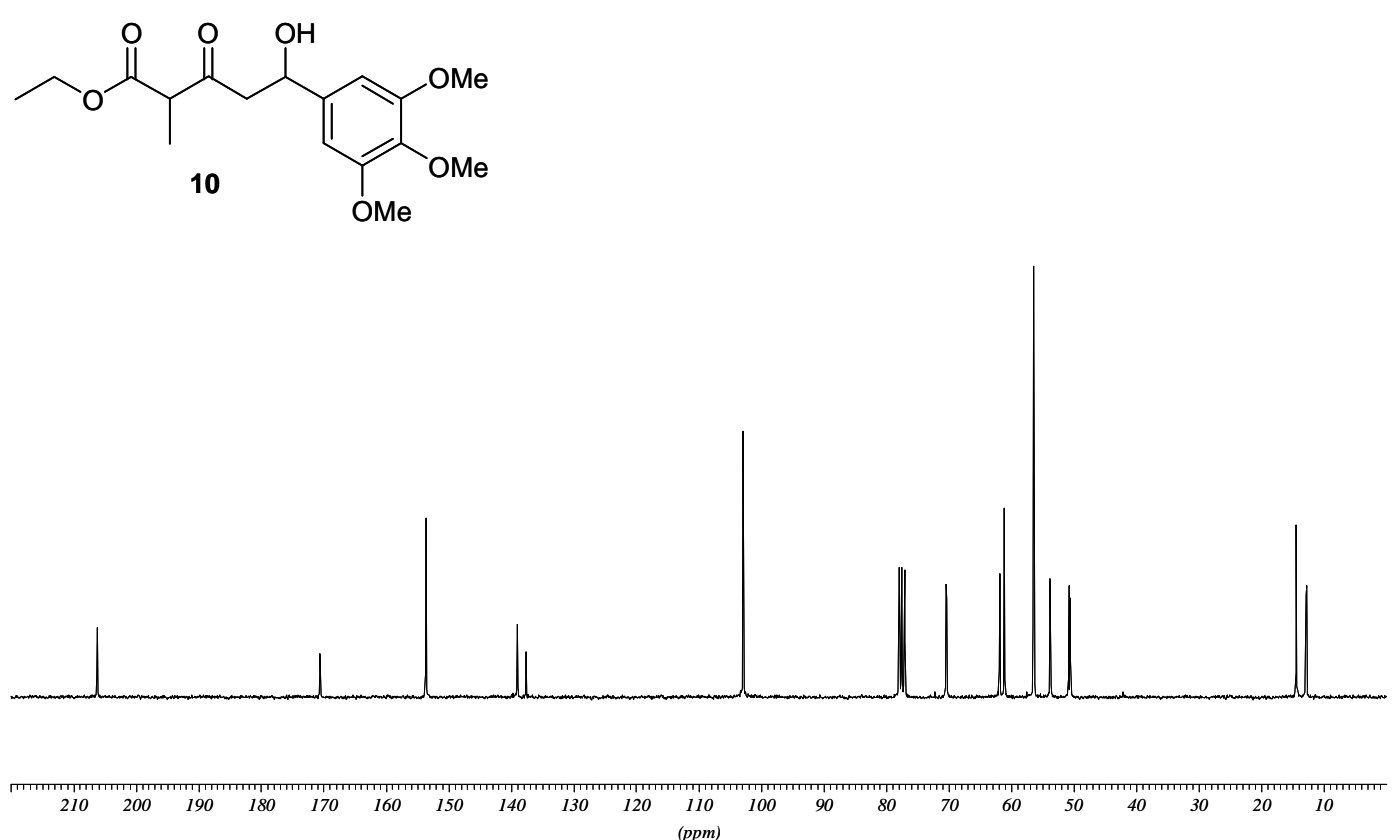
${ }^{1}$ H NMR of benzylidene-(4-methoxy-phenyl)-amine

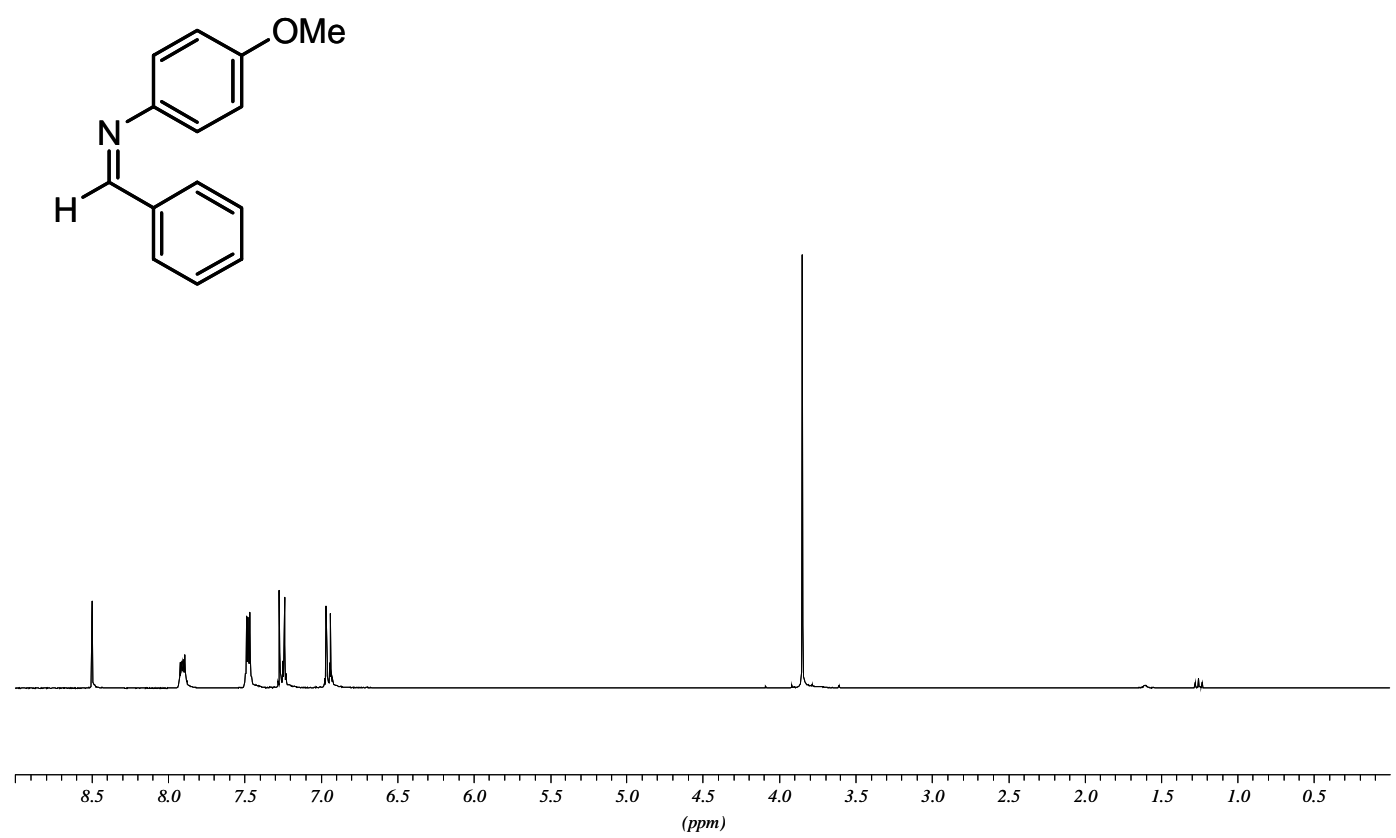

${ }^{13}$ C NMR of benzylidene-(4-methoxy-phenyl)-amine<smiles>COc1ccc(/N=C/c2ccccc2)cc1</smiles>
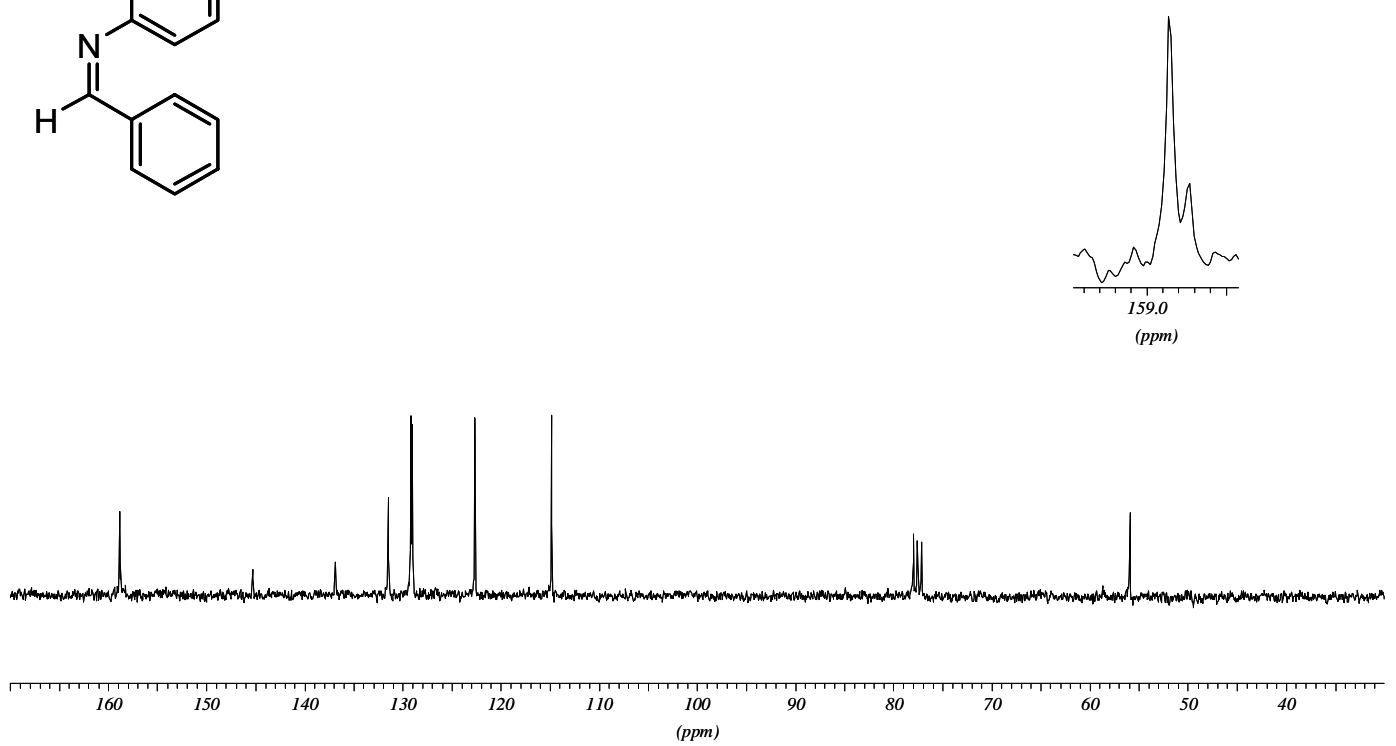


\section{${ }^{1} \mathrm{H}$ NMR of 11}<smiles>COc1ccc(NC(CC(=O)c2ccccc2)c2ccccc2)cc1</smiles>
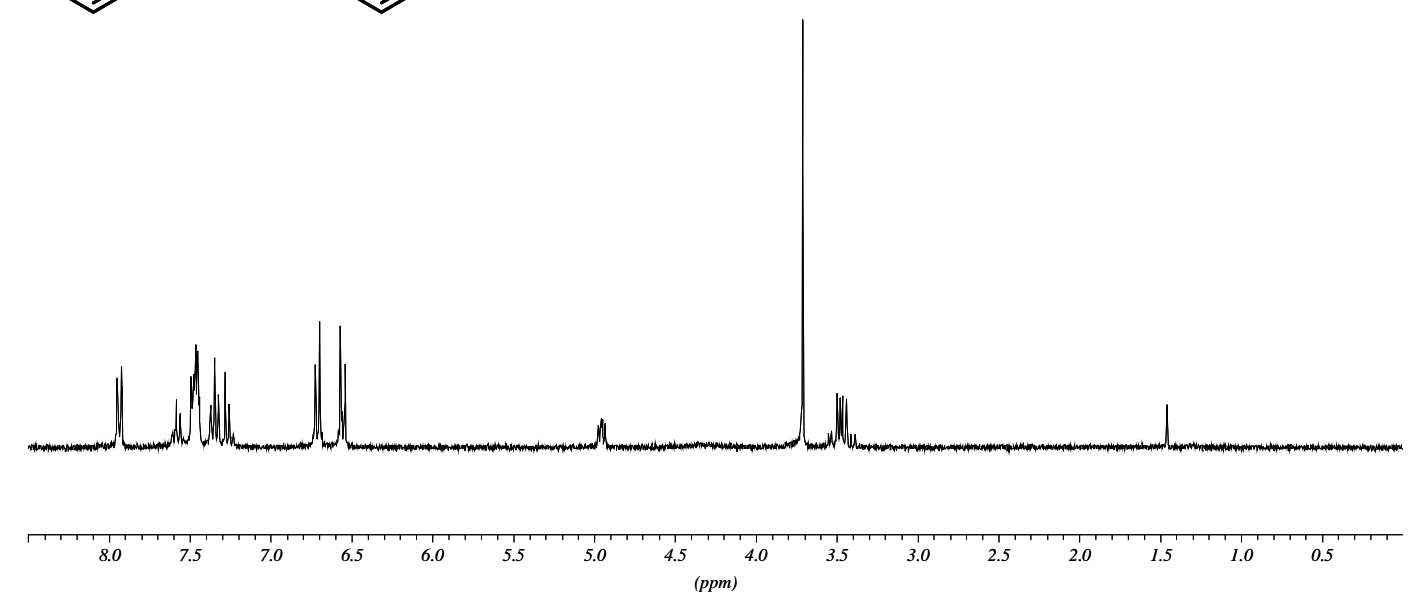

\section{${ }^{13} \mathrm{C}$ NMR of 11}<smiles>COc1ccc(NC(CC(=O)c2ccccc2)c2ccccc2)cc1</smiles>
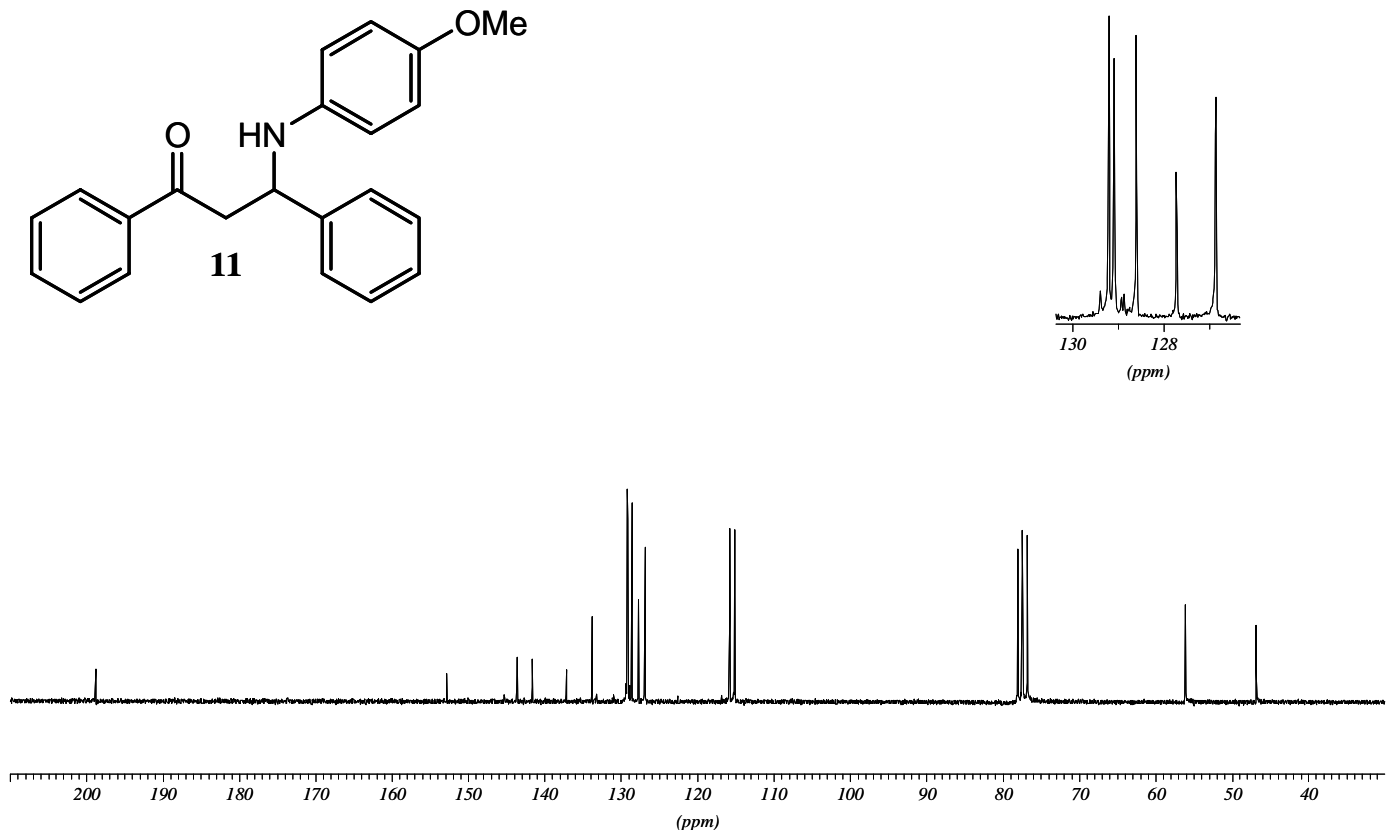
${ }^{1}$ H NMR of $3 w$
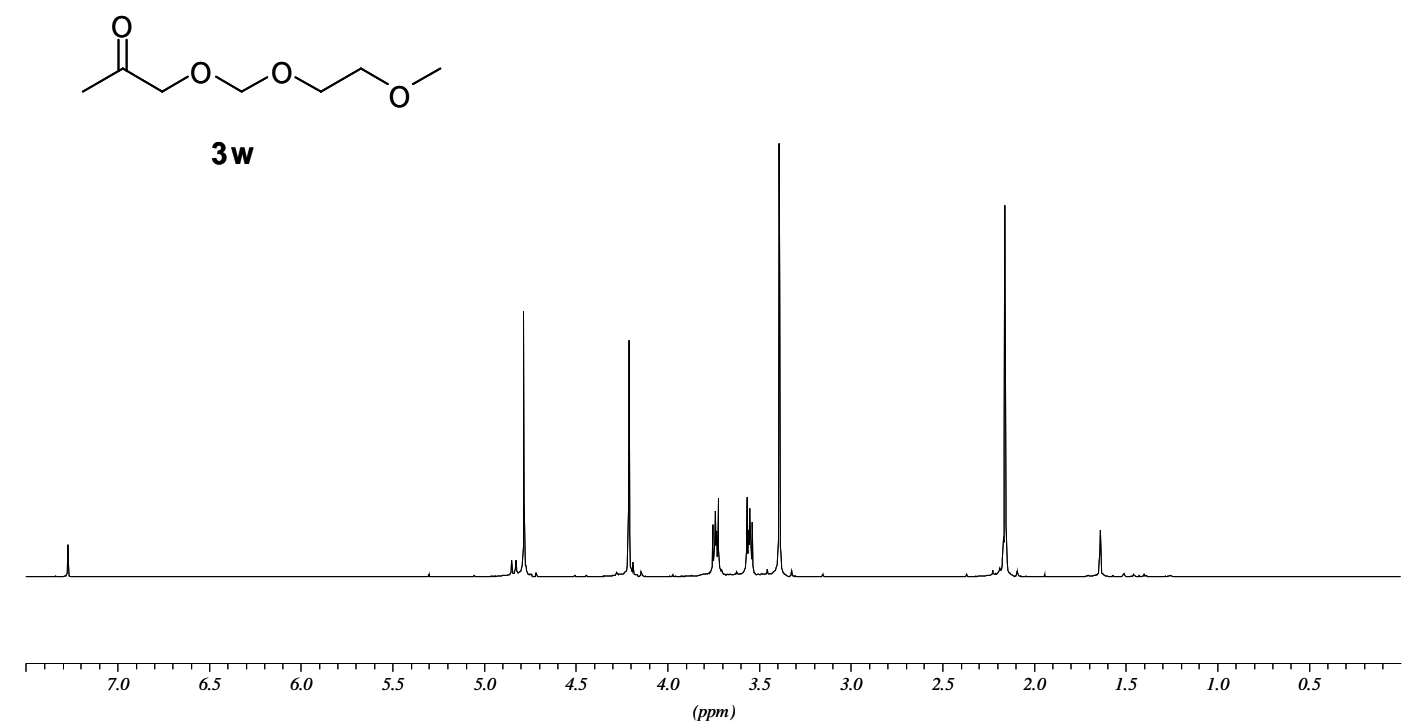

\section{${ }^{13} \mathrm{C}$ NMR of $3 \mathrm{w}$}
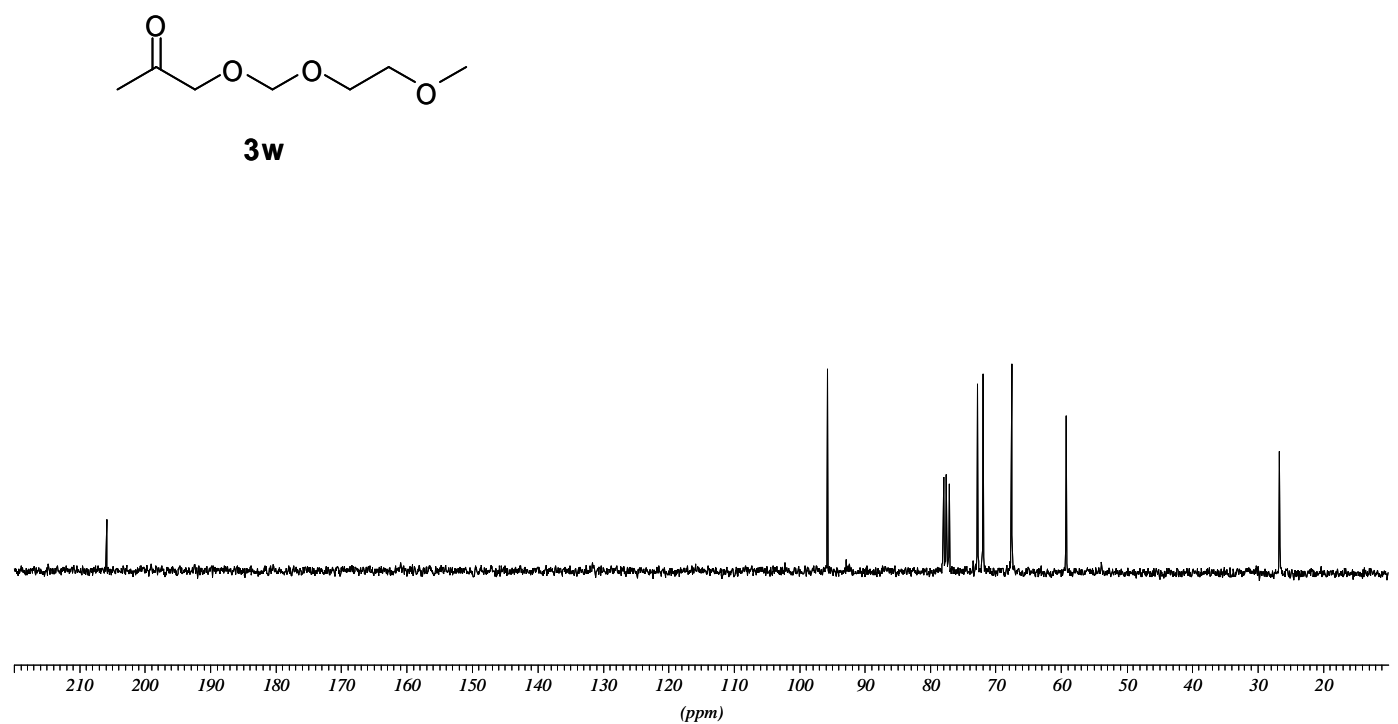